\title{
In vitro analysis of riboflavin-modified, experimental, two-step etch-and-rinse dentin adhesive: Fourier transform infrared spectroscopy and micro-Raman studies
}

\author{
Umer Daood ${ }^{1}$, Chan Swee Heng ${ }^{2}$, Jennifer Neo Chiew Lian ${ }^{3}$ and Amr S Fawzy ${ }^{1}$
}

To modify two-step experimental etch-and-rinse dentin adhesive with different concentrations of riboflavin and to study its effect on the bond strength, degree of conversion, along with resin infiltration within the demineralized dentin substrate, an experimental adhesive-system was modified with different concentrations of riboflavin $(\mathrm{m} / \mathrm{m}, 0,1 \%, 3 \%, 5 \%$ and $10 \%)$. Dentin surfaces were etched with $37 \%$ phosphoric acid, bonded with respective adhesives, restored with restorative composite-resin, and sectioned into resin-dentin slabs and beams to be stored for $24 \mathrm{~h}$ or 9 months in artificial saliva. Micro-tensile bond testing was performed with scanning electron microscopy to analyse the failure of debonded beams. The degree of conversion was evaluated with Fourier transform infrared spectroscopy (FTIR) at different time points along with micro-Raman spectroscopy analysis. Data was analyzed with one-way and two-way analysis of variance followed by Tukey's for pair-wise comparison. Modification with $1 \%$ and $3 \%$ riboflavin increased the micro-tensile bond strength compared to the control at $24 \mathrm{~h}$ and 9-month storage with no significant differences in degree of conversion $(\boldsymbol{P}<0.05)$. The most predominant failure mode was the mixed fracture among all specimens except $10 \%$ riboflavin-modified adhesive specimens where cohesive failure was predominant. Raman analysis revealed that $1 \%$ and $3 \%$ riboflavin adhesives specimens showed relatively higher resin infiltration. The incorporation of riboflavin in the experimental two-step etch-and-rinse adhesive at $3 \%(\mathrm{~m} / \mathrm{m})$ improved the immediate bond strengths and bond durability after 9-month storage in artificial saliva without adversely affecting the degree of conversion of the adhesive monomers and resin infiltration.

International Journal of Oral Science (2014) 7, 110-124; doi:10.1038/ijos.2014.49; published 26 September 2014

Keywords: bond failure; crosslinking; degree of conversion; dentin; experimental adhesive; riboflavin

\section{INTRODUCTION}

Dental adhesion has made marked progress recently as a result of major advances in dentin bonding technology. ${ }^{1}$ The development of bonding agents has increased the clinical consistency of dental composite restorations. ${ }^{2}$ Among the most popular, hybrid layer formation is the most established theory of dentin bonding mechanism. ${ }^{3}$ The chemical and mechanical stability of the collagen fibrils within the hybrid layer is considered to play a role in effective bonding. ${ }^{4}$ However, because of the two-step etch-and-rinse adhesives and larger amounts of hydrophilic monomers, the hybrid layer results in a more porous interface. ${ }^{5}$ Additionally, the phosphoric acid etchant is regarded to have an aggressive effect on the dentin surface, nearly denuding the collagen. ${ }^{6}$ The hybrid layer acts as a permeable membrane resulting in water sorption and collagen degradation at the bottom of the hybrid layer. ${ }^{7}$ The degradation of collagen fibrils is considered to be a likely mechanism for bond deterioration; therefore, it is important to develop a potential strategy for minimising the collagen degradation to improve resin dentin bonding. ${ }^{8}$

Collagen Type I, a predominant variant of collagen types, consists of the $\alpha 1$ and $\alpha 2$ chains within the $\mathrm{N}$-telopeptide, helical and C-telopeptide domains. The central portion of each helical contains the Gly-X-Y sequence, which dominates $95 \%$ of the polypeptide. ${ }^{9}$ The supramolecular arrangement of the fibrillar elements and the microfibrillar networks defines the biophysical characteristics. ${ }^{10}$ Several synthetic and natural chemicals have the ability to increase the number of covalent interand intramolecular collagen crosslinks and are able to affect its properties. ${ }^{11}$ Interestingly, it is well known that the stiffness of the collagen matrix decreases from $18000 \mathrm{MPa}$ in the mineralized state to $1-3 \mathrm{MPa}$ in the demineralized state. This low elasticity modulus permits more rotational and lateral movements of the adjacent collagen peptides, bringing them within reach of the active site of the matrix metalloproteinases (MMPs), ${ }^{12}$ which is a likely phenomenon for dentin collagen degradation. The strengthening of collagen fibrils by crosslinking agents to

${ }^{1}$ Discipline of Oral Sciences, Faculty of Dentistry, National University of Singapore, Singapore, Singapore; ${ }^{2}$ Laboratory technologist, Discipline of Oral Sciences, Biomaterials and Biophotonics, National University of Singapore, Singapore, Singapore and ${ }^{3}$ Discipline of Prosthodontics, Operative Dentistry and Endodontics, National University of Singapore, Singapore, Singapore

Correspondence: Dr AS Fawzy, Discipline of Oral Sciences, Faculty of Dentistry, National University of Singapore, 11 Lower Kent Ridge Road, Singapore 119083, Singapore E-mail: denasfmf@nus.edu.sg

Accepted 3 June 2014 
increase the mechanical properties and decrease the enzymatic degradation may be an important application in restorative dentistry.

The induction of exogenous collagen crosslinks has been proposed as a mechanism for improving the mechanical stability and reducing the collagen biodegradation rates. ${ }^{13}$ Several synthetic (e.g., glutaraldehyde and carbodiimides) and naturally occurring agents (genipin, proanthocyanidin from grape seed extract and others) can induce exogenous collagen crosslinks. ${ }^{14}$ Riboflavin (RF)-catalysed photosensitisation is directly involved in singlet oxygen production, ${ }^{15}$ which results in the involvement of tyrosine residues forming pipi complexes that lead to dityrosine crosslinking. ${ }^{16}$ Previously, proteins have been reported to be pivotal targets for ${ }^{1} \mathrm{O}_{2}$, forging a high rate of chemical reactions involving physical quenching. ${ }^{17}$ Additionally, collagen crosslinking using ultraviolet A (UVA)-activated RF had been successfully reported in the treatment of keratoconus. ${ }^{18-19}$ In an earlier study, we investigated the effect of photo-activated RF on dentin collagen that had increased the biodegradation resistance, the mechanical properties, and the stability of the dentin matrix. ${ }^{20}$ Furthermore, the use of RF as a dentin pre-treatment has been evaluated to strengthen the collagen fibrillar network for optimal dental adhesion. ${ }^{21}$ However, no previous studies have investigated the formulation of RF-modified dentin resin adhesive aimed at improving the durability of resin-dentin bonds and reducing the number of clinical steps required.

Raman spectroscopy is considered to be a useful tool for obtaining structural information ${ }^{22}$ as the resolution limit ( $1 \mu \mathrm{m}$ in diameter), is favourable for evaluating the hybrid layer. ${ }^{23}$ Micro-Raman spectroscopy provides valid information concerning small biochemical changes within the hybrid layer and the extent of resin infiltration. ${ }^{24}$ Additionally, it allows a comprehensive mapping of a defined area as well as the cross section of a hybrid layer. ${ }^{25}$ Furthermore, the vibrational modes not detected within the Fourier transform infrared spectroscopy (FTIR) spectrum are more Raman active. ${ }^{26}$ Therefore, in the present study, micro-Raman spectroscopy offers subsurface imaging without dehydration of the resin dentin specimens.

The aims of this study were to modify a two-step, experimental, etch-and-rinse model dentin adhesive with different concentrations of $\mathrm{RF}$ and to study its effects on the bond strength and the durability of dentin and on the degree of conversion of the experimental adhesives. Additionally, we aimed to determine whether the incorporation of different RF concentrations within the adhesive can improve resin penetration within the demineralized dentin substrate. The null hypotheses are as follows: modification of the experimental adhesives with $\mathrm{RF}$ improves the micro-tensile bond strengths after $24 \mathrm{~h}$ and 9 months of storage in artificial saliva and affects the degree of conversion with the different RF concentrations and has no significant difference in resin infiltration.

\section{MATERIALS AND METHODS}

Sound human molars ( $n=60$; age range: $21-35$ years) were stored after extraction in a solution of deionized water and sodium azide $(0.2 \%)$ at $4{ }^{\circ} \mathrm{C}$ to inhibit microbial growth and were used within 1 month from the start of the experiments. The teeth used in the study were approved by the Institutional Review Board of National University of Singapore. For the resin-bonded specimens, 20 teeth were used for the scanning electron microscope (SEM) analysis; 25 teeth were used for determining microtensile bond strength ( $\mu \mathrm{TBS}$ ); and the remaining 15 teeth were used for the micro-Raman spectroscopy analysis. The chemicals used in this study included riboflavin 5'-monophosphate (RF), 2,2-bis-[4-(2-hydroxy-3methacryloyloxyproxy)phenyl] propane, (Bis-GMA) 2-hydroxyethyl methacrylate (HEMA), ethanol, camphorquinone; ethyl (4-dimethylamino)benzoate and diphenyliodonium hexafluorophosphate (DPIHP, $1.0 \%, \mathrm{~m} / \mathrm{m}$ ) that were purchased from Sigma-Aldrich (St Louis, MO, USA), unless otherwise stated. The two-step Adper ${ }^{\mathrm{TM}}$ Single Bond adhesive was purchased from 3M ESPE (St. Paul, MN, USA).

\section{Preparation of RF-modified experimental resin adhesives}

The experimental two-step model dentin adhesives were prepared as previously described. ${ }^{27}$ The ingredients were weighed using a precision balance (Gold Bell Weigh System, Singapore). The experimental adhesive system is based on bis-GMA and HEMA at a mass ratio of $60: 40$. Ethanol was added as a solvent at a concentration of $30 \%(\mathrm{~m} / \mathrm{m})$. The following three-component visible light photo-initiator systems were used: camphorquinone $(0.5 \%, n / n)$, ethyl (4dimethylamino)benzoate $(0.5 \%, n / n)$ and DPIHP $(1.0 \%, m / m)$. The diphenyliodonium hexafluorophosphate used will act as an electronaccepting initiator. ${ }^{28}$ The concentrations of photo-initiators were calculated according to the total amount of monomers present.

The reactions were conducted in a three-necked flask using a magnetic stirrer (SA300; Sansyo, Tokyo, Japan) and a reflux condenser. The bis-GMA, HEMA and the visible light photo-initiators were added to the ethanol in the flask and were warmed slightly to obtain optimal efficiency in the mixture. To avoid spontaneous polymerisation from the ambient light, the ingredients were kept closed throughout the entire procedure and were mixed under red light in a dark room.

The fabricated model adhesive was left without any modification as a control group or modified using RF of $1.0 \%, 3.0 \%, 5.0 \%$ and $10.0 \%(\mathrm{~m} / \mathrm{m})$ as experimental groups. The powder was added directly to the ethanol solvent with respect to the monomer. The shaking and sonication process helped to yield a well-mixed RF/solvent/monomer solution. After the reaction was completed, the product was centrifuged and complete homogenisation of ingredients and dissolution were confirmed visually. The experimental adhesives prepared were stored at $4{ }^{\circ} \mathrm{C}$ and used within 2 weeks.

\section{Preparation of artificial saliva for specimen storage}

An artificial saliva storage medium was selected to store the specimens for 9 months for additional analysis. The artificial saliva was prepared by dissolving the salts in $900 \mathrm{~mL}$ of $\mathrm{H}_{2} \mathrm{O}$, which was adjusted to $\mathrm{pH} 7.4$ using a diluted solution of $\mathrm{KOH}$. The ingredients included $\mathrm{MgCl}_{2} \cdot 6 \mathrm{H}_{2} \mathrm{O}, \mathrm{CaCl}_{2}$, sodium azide, $\mathrm{KCl}$ and hydroxyethyl-piperazineethane-sulfonic acid (HEPES) buffer, which were added according to the protocol of Hale et al. ${ }^{29}$

\section{Specimen preparation and bonding procedures}

Figure 1 shows the schematic diagram for tooth preparation, bonding, composite build-up, storage time and micro-tensile bond testing. The occlusal enamel of the molars was cut perpendicular to the long axis of the teeth using a low-speed, water-cooled, diamond saw (Buehler, Lake Bluff, IL, USA) until the dentin surface was exposed $1 \mathrm{~mm}$ below the dentino-enamel junction (DEJ). The dentin appeared yellow with minimal evidence of translucency. ${ }^{30}$

The double-sided adhesive tape $(0.10 \mathrm{~mm}$ thick $)$ with a $5.0-\mathrm{mm}$ opening was attached to the dentin surface to approximate the bonding area. A transparent acrylic tube of similar diameter and of $5 \mathrm{~mm}$ in height was placed on the peeled adhesive tape, exposing the dentin surface that was etched using $37 \%$ phosphoric acid gel (3M ESPE Scotchbond ${ }^{\mathrm{TM}}$, St. Paul, MN, USA) for $15 \mathrm{~s}$, and then rinsed with deionized water for $20 \mathrm{~s}$. The excess water was removed from the surface using a damp cotton pellet leaving the dentin surface visibly moist. Two consecutive coats, each prepared using experimental dentin adhesive, were applied to the dentin surface for $15 \mathrm{~s}$ using a 


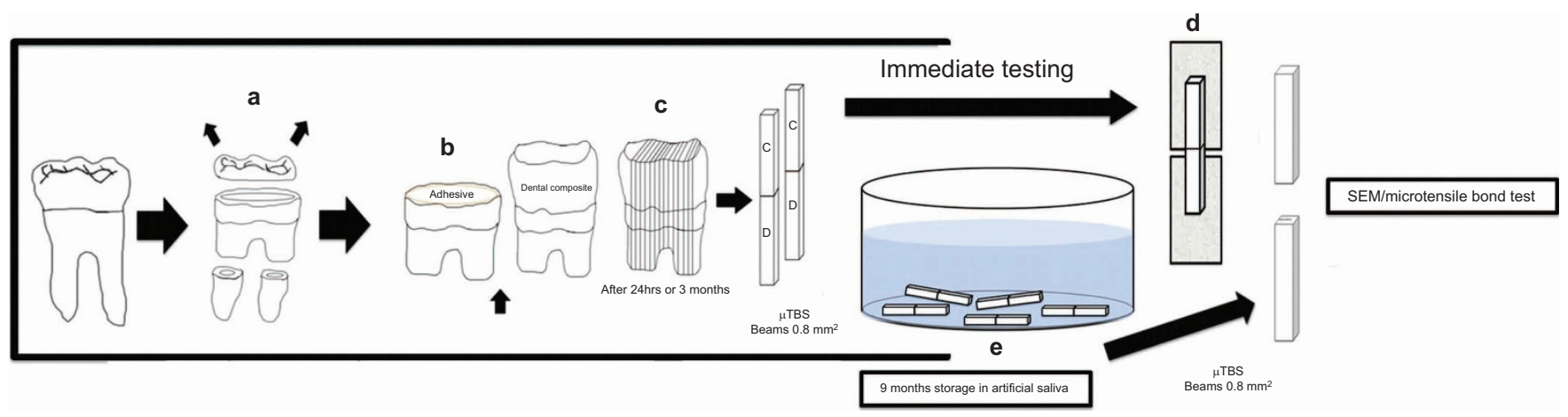

Figure 1 Schematic representation of the specimen preparation for the $\mu$ TBS test and SEM analysis. (a) Removal of occlusal surface to expose the flat dentin surface; (b) preparation of the dentin surface to receive adhesive with or without RF; (c) cutting of resin-dentin beams from the center of bonded specimen; (d) attachment of the single resin-dentin beam for immediate $\mu$ TBS testing; (e) storage of resin-dentin beams in artificial saliva for 9-months for $\mu$ TBS testing and SEM analysis. $\mu \mathrm{TBS}$, micro-tensile bond strength; RF, riboflavin.

micro-brush passively and gently air-thinned for $5 \mathrm{~s}$ to remove the excess solvent. A magnifying lens was used to ensure that all of the bonding surfaces were covered with the adhesives before polymerisation. Photo-polymerisation was conducted at room temperature using a dental blue light curing unit (Curing Light 2500; 3M ESPE, St. Paul, $\mathrm{MN}, \mathrm{USA})$ for $20 \mathrm{~s}$ at a $10-\mathrm{mm}$ distance from the surface with $600 \mathrm{~mW} \cdot \mathrm{cm}^{-2}$ output. The resin composite (Filtek ${ }^{\mathrm{TM}} \mathrm{Z} 350 \mathrm{XT}$; $3 \mathrm{M}$ ESPE, St. Paul, MN, USA) was applied in four increments. The first increment was applied in less than $0.5 \mathrm{~mm}$ thick in the acrylic tube to reduce the effect of polymerisation shrinkage. All of the increments were light cured for $20 \mathrm{~s}$. The specimens were stored in artificial saliva for $24 \mathrm{~h}$ at $37{ }^{\circ} \mathrm{C}$ to complete the polymerisation reaction. The specimens were used for immediate SEM and micro-Raman analyses. Additionally, several specimens were stored in artificial saliva for 9 months for SEM evaluation after the ageing period.

\section{SEM observation of resin/dentin interface}

The specimens were sectioned perpendicular to the bonded surface to expose the adhesive interface to obtain 1-mm thick resin-dentin slabs. From each tooth, 2-3 slabs were obtained from the mid-coronal dentin. The resin-dentin slabs were wet-polished with increasing grit sizes (600-2 500) of SiC papers (Carbimet, Buehler, Lake Bluff, IL, USA) and rinsed with distilled water for $10 \mathrm{~min}$ under ultrasonic agitation. The polished surfaces were dried by gentle blotting using absorbent paper (Kimwipes; Kimberly-Clark Professional, Roswell, GA, USA). The slabs from each group were randomly subdivided, to be viewed in artificial saliva after $24 \mathrm{~h}$ ( $n=5$ for each group) or 9 months of storage ( $n=5$ for each group).

The resin-dentin slabs were etched with $35 \%$ phosphoric acid gel for $15 \mathrm{~s}$, rinsed with distilled water for $15 \mathrm{~s}$, air-dried, submerged in $5.25 \%$ sodium hypochlorite solution for $20 \mathrm{~min}$ and thoroughly washed with distilled water for $5 \mathrm{~min}$. The specimens were placed in ascending grades of 50\%,75\%,80\%, 95\% and $100 \%$ ethanol for sequential drying before being desiccated in critical point drying (Balzers 030, Shimadzu, Kyoto, Japan). The specimens were then mounted on aluminium stubs using conductive tape (double-sided carbon tape) and then gold sputtered for $120 \mathrm{~s}$ (Baltec SCD sputter, Scotia, NY, USA). After gold sputtering, the specimens were viewed using SEM (Philips/FEI XL30 FEG SEM, Hillsboro, OR, USA), operated at an accelerating voltage of $10 \mathrm{kV}$ at different magnifications.

\section{$\mu \mathrm{TBS}$}

The restored teeth ( $n=25$; five in each group) were sectioned using a low-speed, water-cooled, diamond saw (Buehler, Lake Bluff, IL, USA) into several $1 \mathrm{~mm} \times 1 \mathrm{~mm}$ resin-dentin beams with resin composite forming the upper half of the beam and the underlying dentin forming the lower half of the beam. The beams in each group were randomly divided into groups to be stored in artificial saliva for $24 \mathrm{~h}$ or 9 months $(n=27$ each). The prematurely debonded beams were also recorded per tooth. For $\mu$ TBS testing, each beam was fixed to a custommade metallic jig mounted to a universal testing machine (Instron 5848, Microtester) using cyanoacrylate adhesive (Zapit; Dental Ventures of America, Corona, CA, USA). A tensile load was applied at a crosshead speed of $0.5 \mathrm{~mm} \cdot \mathrm{min}^{-1}$ until failure was achieved. Subsequently, the specimens were carefully removed using a scalpel blade, and the crosssectional area was measured at the site of fracture to the nearest $0.01 \mathrm{~mm}$ using a digital calliper (Model 500-196-20, Mitutoyo Digimatic Caliper) for calculating $\mu \mathrm{TBS}$ expressed in $\mathrm{MPa}$.

\section{Fractographic analysis using SEM}

From each group, 15 fractured beams from $\mu$ TBS testing were prepared and viewed using a stereomicroscope (Olympus, Tokyo, Japan) at $\times 30$ and $\times 100$ magnifications to separate the different fractured surfaces and to evaluate the fracture pattern before SEM for the fractographic analysis. The fractured resin and the dentin sides of each beam were air-dried, secured on aluminium stubs using conductive tape (double-sided carbon tape) and sputter coated using gold palladium alloy (30 nm, Bal-Tec SCD 005, Sputter Coater) for $120 \mathrm{~s}$. After gold sputtering, the specimens were viewed using SEM (Philips/FEI XL30 FEG SEM, Hillsboro, OR, USA), operated at an accelerating voltage of $10 \mathrm{kV}$ at different magnifications. The areas of the fractured sites were expressed as percentages of the fractured specimens and were calculated from the SEM microphotographs using an image analyser (Digitizer, Graphtec, Tokyo, Japan). The fractographic analysis was performed for four representative debonded beams stored in artificial saliva for $24 \mathrm{~h}$ and 9 months. The failure modes of each resindentin bonds were categorized as follows: (M) mixed failure, (A) cohesive failure in adhesive, $(\mathrm{CD})$ cohesive in dentin and (CC) cohesive in resin composite. ${ }^{31}$

\section{Degree of conversion}

The degree of conversion (DC) of the experimental adhesives was measured using transmission FTIR. For the FTIR analysis of the un-modified and RF-modified bonding agents, $15 \mu \mathrm{L}$ of adhesive was placed in a well before testing. The concentration of available carbon-carbon double bonds in the experimental adhesives were measured and the absorbance peaks were recorded by transmission mode; a drop of adhesive was smeared onto the potassium bromide discs 
$(2.0 \times 2.0)$ (Shimadzu, Kyoto, Japan) at a resolution of $4 \mathrm{~cm}^{-1}$. The adhesive was left undisturbed before curing and air-thinned with a gentle stream of oil-free air. During the spectrum analysis, the uncured resin was continuously in contact with the sensor. The absorbance peaks for the uncured specimens were recorded using the FTIR spectrometer (FTIR-8800; Shimadzu, Kyoto, Japan); the DC was determined by obtaining a spectrum ranging between 400 and $4000 \mathrm{~cm}^{-1}$ (30 scans). After recording the absorbance peaks in uncured resin, the adhesives were photo-cured and the FTIR was recorded again at different time points (starting from $10 \mathrm{~s}$ until $30 \mathrm{~min}$ ) from the start of irradiation. The $\mathrm{C}=\mathrm{C}$ absorbance peak at $1638 \mathrm{~cm}^{-1}$ (unpolymerized methacrylate stretching vibration) and the $\mathrm{C}-\mathrm{C}$ reference peak at $1607 \mathrm{~cm}^{-1}$ (stretching vibration of the aromatic ring $)^{32}$ were obtained using the baseline technique. ${ }^{33}$ The ratio of $(\mathrm{C}=\mathrm{C}) /(\mathrm{C}-\mathrm{C})$ absorbance intensities (percentage of unreacted double bonds) was measured before and after polymerisation as shown in Equation (1): $:^{34}$

$$
\mathrm{DC}=\left[1-\left(C_{\text {aliphatic }} / C_{\text {aromatic }}\right) /\left(U_{\text {aliphatic }} / U_{\text {aromatic }}\right)\right] \times 100 \%
$$

where $C_{\text {aliphatic }}$ is the absorption peak at $1638 \mathrm{~cm}^{-1}$ of the polymerized resin, $C_{\text {aromatic }}$ is the absorption peak at $1607 \mathrm{~cm}^{-1}$ of the polymerized resin, $U_{\text {aliphatic }}$ is the absorption peak at $1638 \mathrm{~cm}^{-1}$ of the unpolymerized resin and $U_{\text {aromatic }}$ is the absorption peak at $1607 \mathrm{~cm}^{-1}$ of the unpolymerized resin.

All of the experiments were performed in triplicate to determine important variations in our technique that might have a minimum number for a standard deviation.

\section{Micro-Raman spectroscopy of resin-dentin specimens}

The Raman spectra were recorded on a micro-Raman using a JY LabRam HR 800 Raman spectrometer (Horiba Jobin Yvon, Longjumeau, France) equipped with Leica lenses and curve-fitting Raman microscopic software (Labspec 5). The visual identification of the position at which the Raman signal was obtained from the adhesive interface was adjusted accordingly and background-corrected for dark counts with intensity-normalized. Zero calibration was performed and the 785-nm single model laser was focused onto the selected resin/dentin adhesive interface area at a power of $<500 \mu \mathrm{W}$ using 100X/NA 0.9 objective lens. The spectral information was obtained between $700 \mathrm{~cm}^{-1}$ and $1800 \mathrm{~cm}^{-1}$ and the spectra were filtered to remove noise. With a laser spot diameter of $1 \mu \mathrm{m}$ and resindentin specimen placed on a glass slide, Raman scattering was induced at different locations within the adhesive interface using a computer-controlled $x-y-z$ stage. The specimens of dentin and cured commercially available Adper ${ }^{\mathrm{TM}}$ Single Bond two-step etch-and-rinse adhesive (3M ESPE, St. Paul, MN, USA) were also analysed for the generation of standard spectra (Figure 2). The exposure time on each scan was $60 \mathrm{~s}$.

The line scans across each resin/dentin specimens were obtained in the regions of the bonded interface starting in the dental composite region (on the left), across the resin/dentin adhesive interface and ending onto the dentin side (on the right). The scans were acquired in $1-\mu \mathrm{m}$ steps using the $x-y-z$ stage and obtained over the spectral regions. The measurements were performed three times for each specimen to confirm the reproducibility of the technique. The peak intensity at $960 \mathrm{~cm}^{-1}$, which is representative of the mineral content of normal dentin by $\mathrm{PO}_{4}$ vibration, $1659-1667 \mathrm{~cm}^{-1}$ and 1243 $1246 \mathrm{~cm}^{-1}$, which are representative of the organic content of dentin by Amide I and III, ${ }^{35}$ respectively, were obtained as standards (Figure 2), for comparing the readings of the control and RF-modified adhesive specimens after resin infiltration and bonding. The peak at $1640 \mathrm{~cm}^{-1}$ is related to the $\mathrm{C}=\mathrm{C}$ methacrylate groups of bis-GMA and
Table 1 Typical band assignments of Raman spectrum of dentin collagen and Single Bond

\begin{tabular}{lcl}
\hline & \multicolumn{2}{c}{ Spectral identification } \\
\cline { 2 - 3 } Product & \multicolumn{1}{c}{ Raman shift/cm $\mathrm{cm}^{-1}$} & \multicolumn{1}{c}{ Raman assignment } \\
\hline Single bond & 113 & \multicolumn{1}{c}{ Phenyl, C-O-C } \\
& 1230 & $\mathrm{CH}-\mathrm{OH}$ stretch \\
& 1404 & $\mathrm{CH}-\mathrm{OH}, \mathrm{CH}_{2}-\mathrm{OH}$ stretch \\
& 1452 & $\mathrm{CH}_{2}, \mathrm{CH}_{3}$ deformation \\
& 1720 & $>\mathrm{C}=\mathrm{O}$ \\
Dentin & 1246 & $\mathrm{Amide} \mathrm{III}$ \\
& 1450 & $\mathrm{CH}_{2}$ \\
& 1667 & $\mathrm{Amide} \mathrm{I}$ \\
\hline
\end{tabular}

HEMA monomers. Using complete polymerisation, the $\mathrm{C}=\mathrm{C}$ is converted to $\mathrm{C}-\mathrm{C}$ to form the polymer chain. The spectral identification for Single Bond and dentin are presented in Table 1.

\section{Statistical analysis}

The data are expressed as the mean \pm standard deviation values and analysed using one-way and two-way analyses of variance (ANOVA) followed by Tukey's test for pair-wise multiple comparison between groups. $P<0.05$ is considered to be statistically significant.

\section{RESULTS}

\section{SEM analysis of resin/dentin interface}

Figures 3 show an overall view of the resin/dentin interfaces of the specimens treated with and without RF-modified experimental adhesives after $24 \mathrm{~h}$ and 9 months of storage time, respectively. The interdiffusion or hybrid layer along with resin tags is noted in all of the bonded interfaces in which the experimental adhesive systems were used. The thickness of the hybrid layer and the length of the resin tags showed a visible variation according to the type of adhesive used.

After $24 \mathrm{~h}$ of storage in artificial saliva, the sizeable resin tags with numerous lateral branches and a well-defined intact hybrid layer are highlighted along with the control and 1\% RF-modified adhesive specimens (Figure $3 \mathrm{a}$ and $3 \mathrm{~b}$ ). The specimens for the 3\% RF-modified adhesives showed a well-organized homogeneous hybrid layer (Figure 3c) and numerous well-developed resin tags. Additionally, the adhesive interface with the overlying composite resin was found to be intact and without any voids. The resin/dentin interface of the 5\% RFmodified adhesive revealed a thick and textured hybrid layer with long well-formed resin tags (Figure 3d). The 10\% RF-modified adhesive demonstrated shorter resin tags and a thinner hybrid layer (Figure 3e).

The changes observed in the SEM findings after 9 months of storage in artificial saliva were dissimilar to the SEM findings after a 24-h storage. Intact hybrid layer could be observed after a 9-month storage for the control, $1 \%$ and 3\% RF-modified specimens (Figure $3 \mathrm{f}-3 \mathrm{~h}$ ) compared to the 5\% RF-modified specimens (Figure 3i). Hardly any hybrid layer could be observed in the $10 \%$ RF-modified specimens and only few short resin tags could be observed (Figure $3 \mathrm{j}$ ).

\section{Micro-tensile bond strength and fractographic analysis}

The results of $\mu$ TBS testing are summarized in Table 2 . The two-way ANOVA showed a significant effect and interaction $(P<0.05)$ of the independent variables (RF concentration and storage time in artificial saliva) on the $\mu$ TBS. The $3 \%$ RF-modified group showed the highest $\mu \mathrm{TBS}$ compared to the control and all other experimental groups after a 24-h storage in artificial saliva. No difference was found between the control and 1\% RF-modified specimens in the baseline measurements 

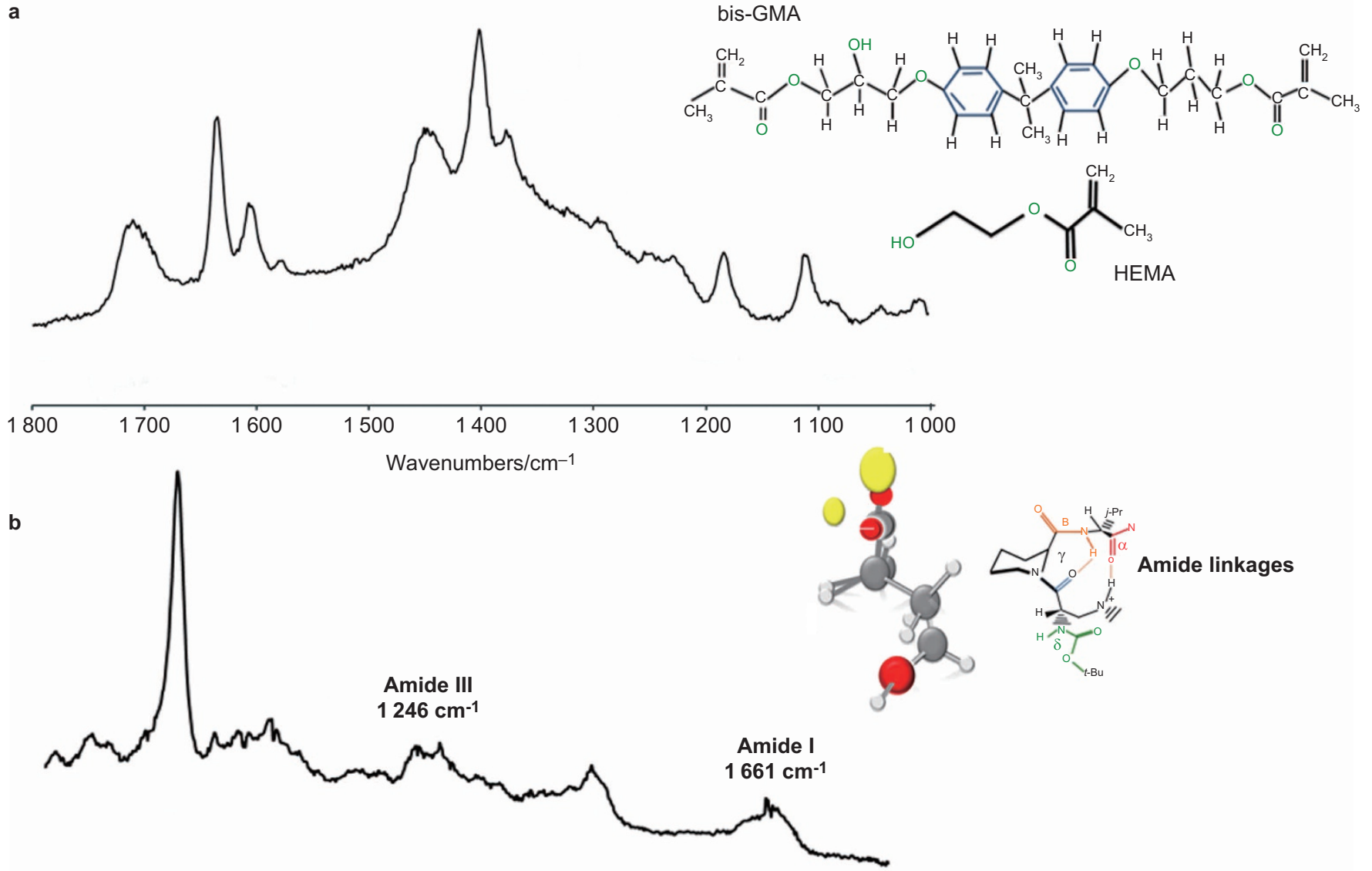

$\begin{array}{rlllllllll}800 \quad 900 & 1000 & 1100 & \begin{array}{c}1200 \\ \text { Wavenumbers } / \mathrm{cm}^{-1}\end{array} & 1300 & 1400 & 1500 & 1600 & 1700 & 1800\end{array}$

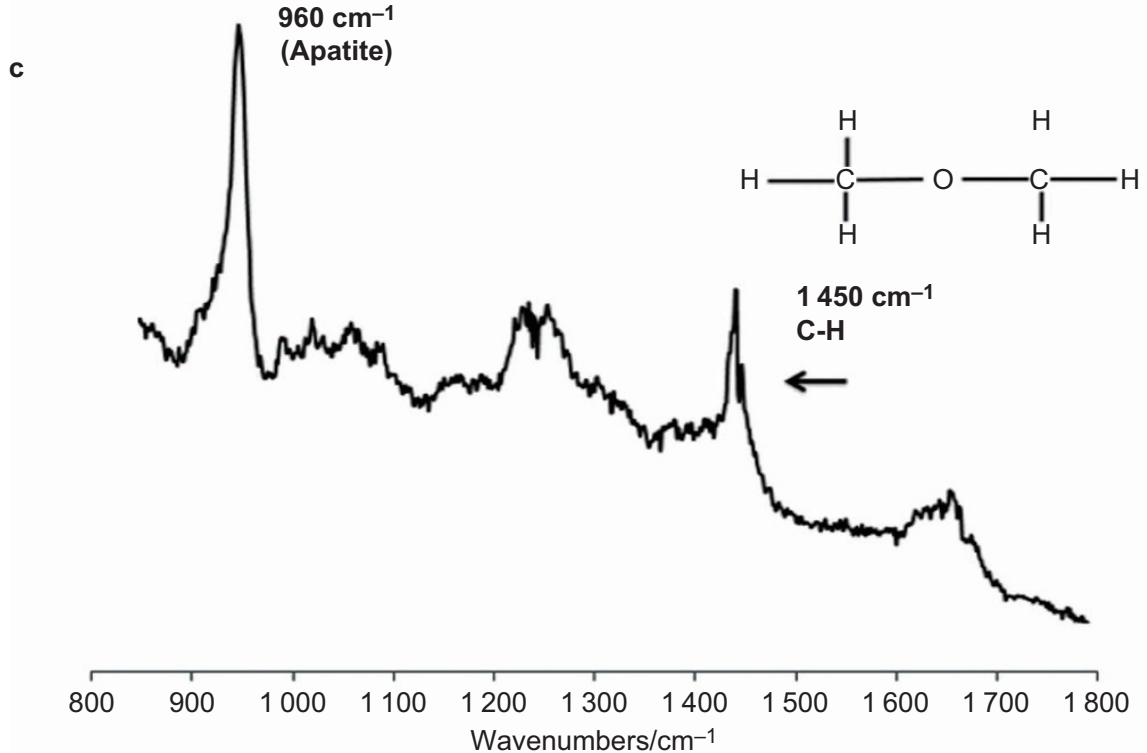

Figure 2 Representative Raman spectra recorded in the region between 800 and $1800 \mathrm{~cm}^{-\mathbf{1}}$. (a) Adper ${ }^{\mathrm{TM}}$ Single Bond adhesive; (b) dentin specimen; (c) resin impregnated dentin. The $\mathrm{P}-\mathrm{O}$ bond at $960 \mathrm{~cm}^{-1}$ of the mineral component is well represented. The peaks at $1667 \mathrm{~cm}^{-1}$ and $1246 \mathrm{~cm}^{-1}$ are associated with organic components for dentin collagen. bis-GMA, 2,2-bis-[4-(2-hydroxy-3-methacryloyloxyproxy)phenyl] propane; HEMA, 2-hydroxyethyl methacrylate. 

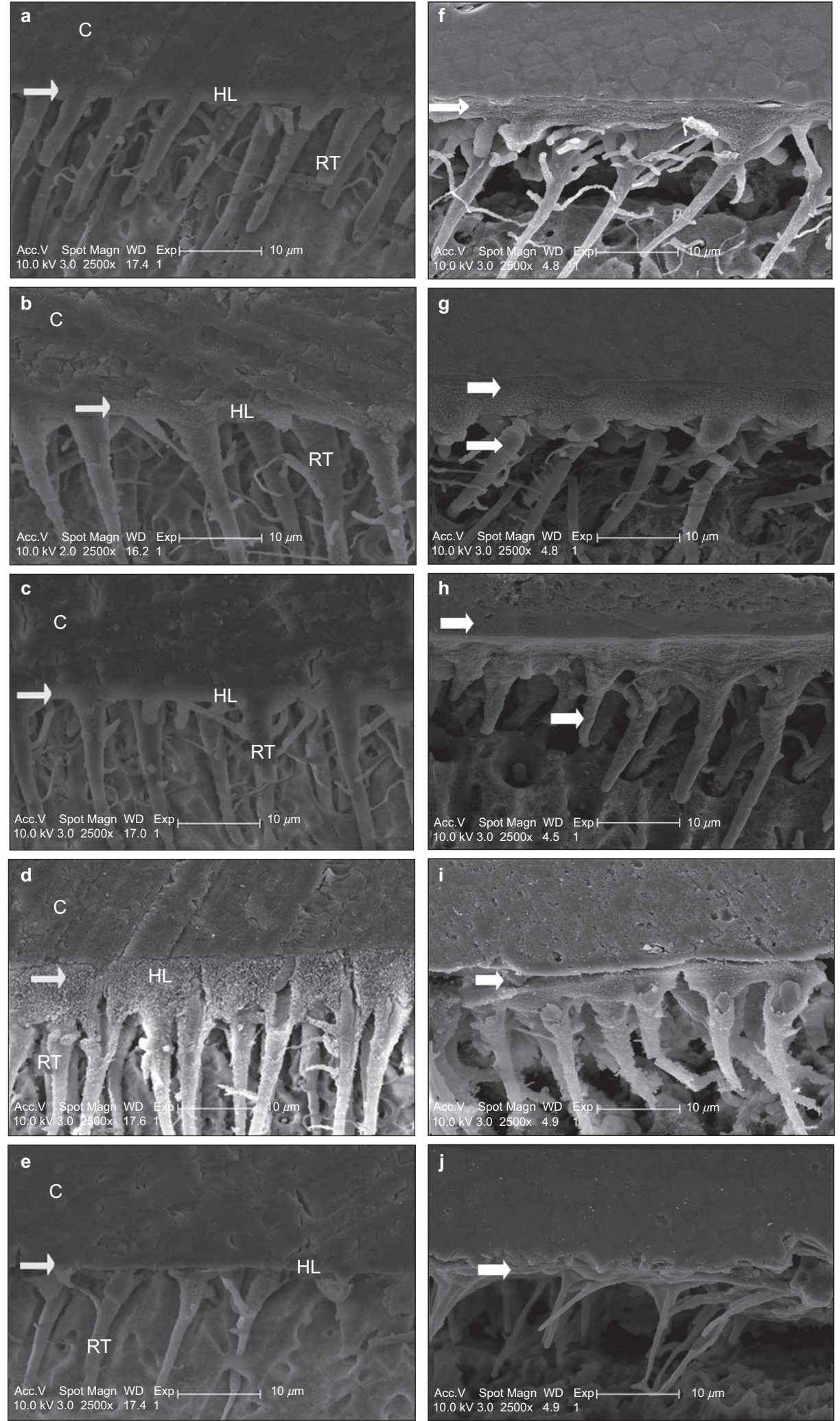

Figure 3 SEM images of the resin-dentin interface after 24-h and 9-months storage in artificial saliva. (a-e) 24-h storage; (f-j) 9-months storage. Well-defined uniform hybrid layer and with well-formed branched resin tags could be observed with control (a), 1\% RF-modified adhesive (b), and 3\% RF-modified adhesive (c) specimens. (d) Relatively thick and textured hybrid layer with long well formed resin tags could be seen with the 5\% RF modified adhesive specimens. (e) The $10 \%$ RFmodified adhesive specimens showed a very thin hybrid layer with lack of well-formed resin tags. Relatively intact hybrid layer could be seen after 9 -months storage for the control (f), $1 \%(\mathbf{g})$ and 3\% RF-modified specimens (h) compared to the 5\% RF-modified specimens (i). (j) Hardly any hybrid layer could be observed in the 10\% RF-modified specimens and only few short resin tags could be seen. White arrow pointing towards hybrid layer. C: resin composite; HL: hybrid layer; RT: resin tags; SEM, scanning electron microscope. 
Table 2 Estimated $\mu$ TBS of adhesives after $24 \mathrm{~h}$ and 9 months

\begin{tabular}{lccc}
\hline & & \multicolumn{2}{c}{$\mu \mathrm{TBS} / \mathrm{MPa}$} \\
\cline { 3 - 4 } Experimental modified adhesives & RF concentration/\% & $24 \mathrm{~h}$ & 9 months \\
\hline I (control) & 0 & $37.1( \pm 6.8)^{\mathrm{ABa}}$ & $25.5( \pm 7.2)^{\mathrm{Ab}}$ \\
II & 1 & $38.8( \pm 7.5)^{\mathrm{Ba}}$ & $33.2( \pm 6.8)^{\mathrm{Bb}}$ \\
III & 3 & $44.4( \pm 8.6)^{\mathrm{Ca}}$ & $37.3( \pm 7.4)^{\mathrm{Cb}}$ \\
IV & 5 & $33.6( \pm 7.7)^{\mathrm{Aa}}$ & $26.9( \pm 6.1)^{\mathrm{Ab}}$ \\
V & 10 & $25.1( \pm 8.1)^{\mathrm{Da}}$ & $18.7( \pm 7.3)^{\mathrm{Db}}$ \\
\hline
\end{tabular}

$\mu$ TBS, micro-tensile bond strength; RF, riboflavin.

Numbers are means and values in brackets are standard deviations. The superscript letters indicate statistically significant differences within the columns and rows $(P<0.05)$. $n=27$ for each group. Test area $1.0 \mathrm{~mm}^{2}$.

at $24 \mathrm{~h}$. In contrast, the $10 \%$ RF-modified specimens showed the lowest $\mu \mathrm{TBS}$ compared to all other groups.

Storing the resin-dentin beams for 9 months in artificial saliva significantly decreased the $\mu$ TBS within each of the control and the RF-modified groups. Only $1 \%$ and 3\% RF-modified groups showed significantly higher $\mu$ TBS after a 9-month storage compared to the control group. However, no difference was found between the control group and the 5\% RF-modified groups. As for the 24-h storage, the $10 \%$ RF-modified group showed the least $\mu$ TBS after a 9-month storage.
The percentage of failure mode distribution is shown in Figure 4. For all specimens tested after both 24-h and 9-month storage, the mixed failure mode was predominant except for the $10 \%$ RF-modified group, where the cohesive failure in the adhesive was the predominant failure mode. However, no cohesive failure in dentin was found in the $10 \%$ RF-modified specimens after a 9-month storage.

Figure 5 shows selected SEM images of the fractured surfaces of the resin-dentin beams after 24 -h and 9-month storage in artificial saliva. In the 24-h specimens, failure occurred for the most part at the bottom

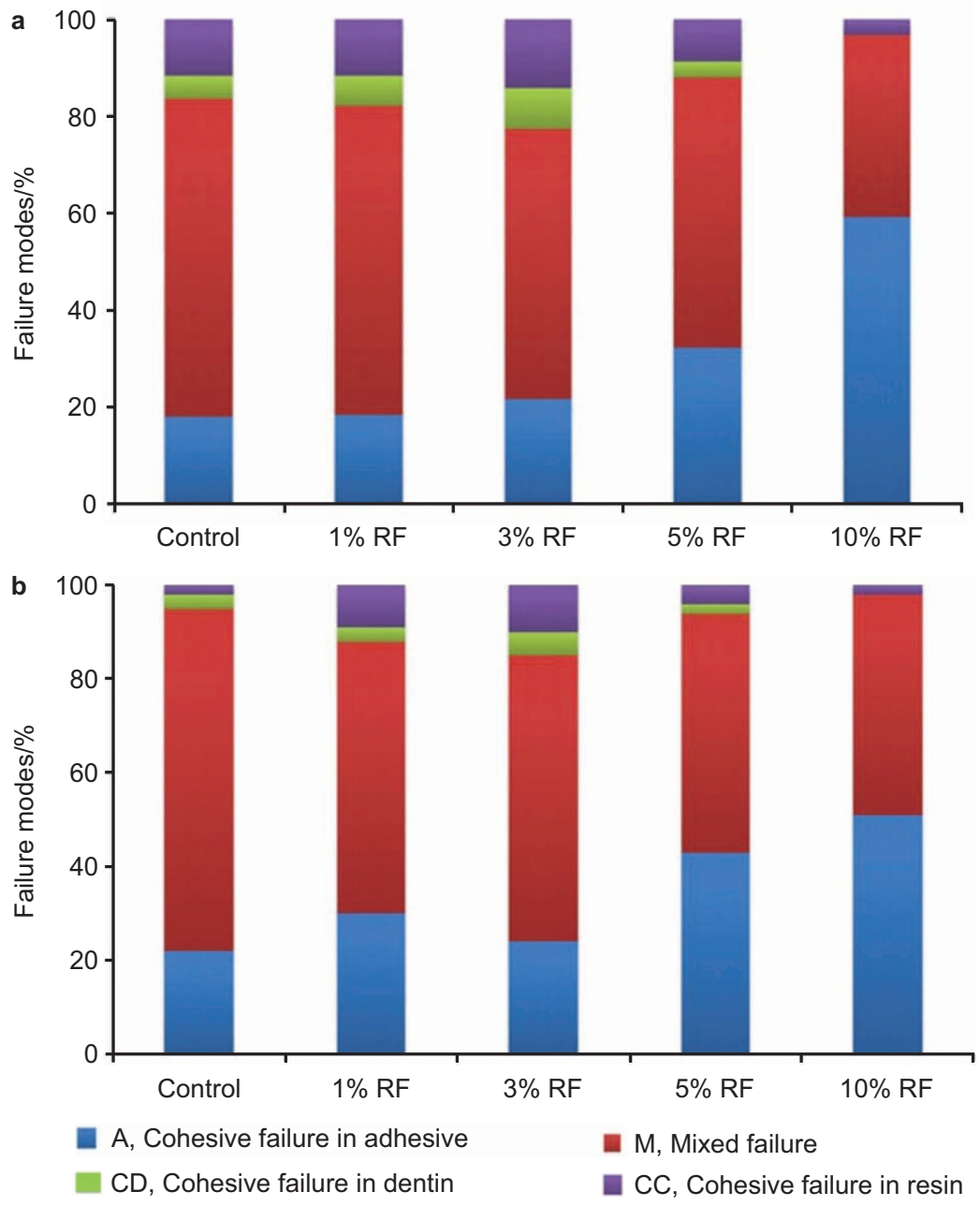

Figure 4 Distribution of failure mode of control and RF-modified adhesive specimens after the micro-tensile strength testing. (a) 24 h; (b) 9 - months storage in artificial saliva. RF, riboflavin. 

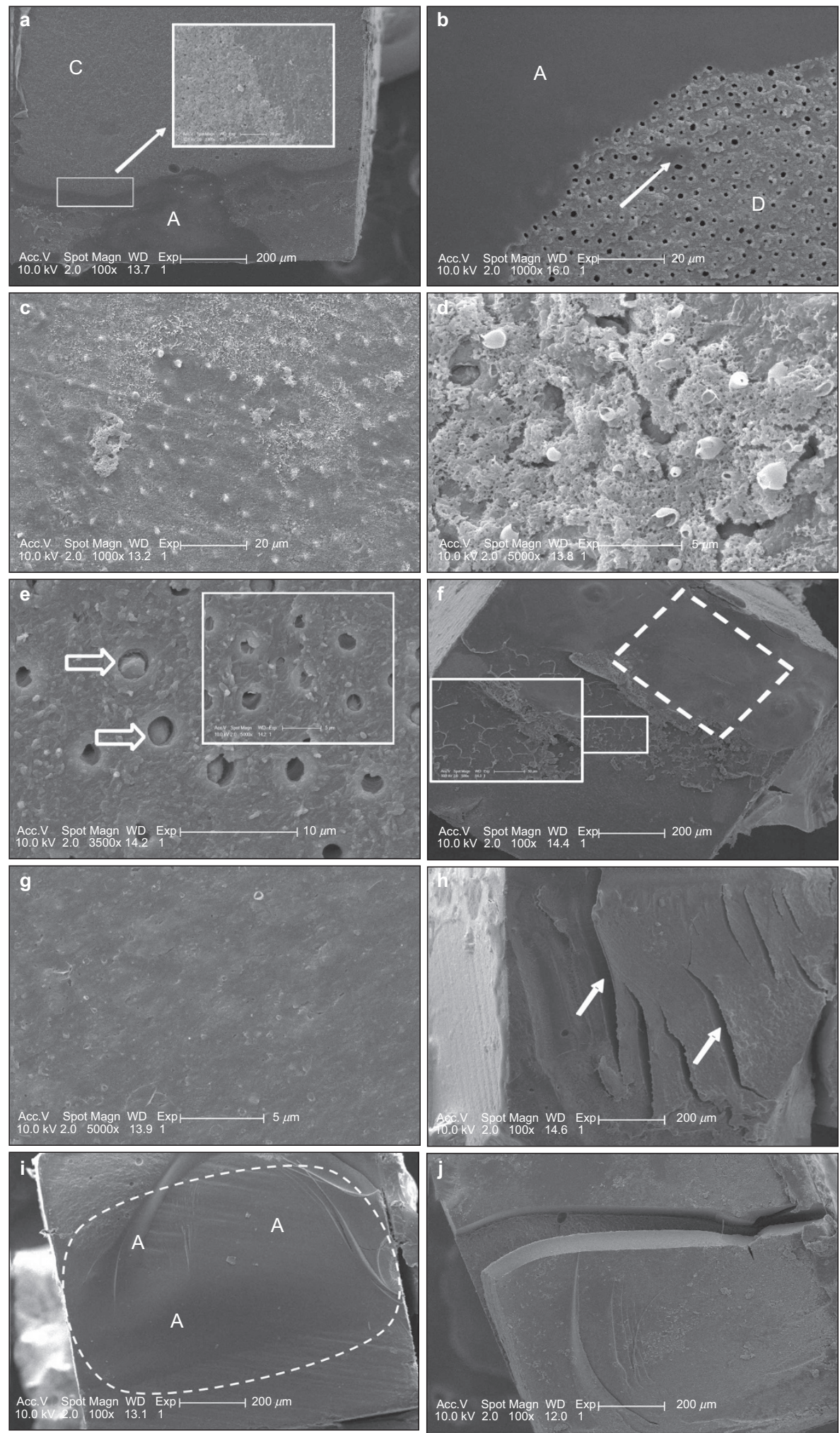

Figure 5 Representative fracture surfaces of specimens bonded with control and RF-modified adhesives. (a) Control group, without RF, showing a typical mixed fracture pattern at the outer rim, occurring mostly at the bottom of the hybrid layer (higher magnification shown in solid box). (b) mixed failure in 1\% RF-modified adhesive specimens with open dentinal tubules and adhesive remnants (arrow). (c and d) 3\% RF-modified adhesive specimens, with mixed failure pattern and with resin tags. (e) Resin tags within the dentinal tubules of 3\% RF-modified adhesive specimens (open arrows). (f) $5 \%$ RF-modified adhesive specimens at lower magnification presenting dentin side of fracture with mixed failure pattern (solid box) and cohesive failure within the adhesive (dotted box). (g) Adhesive failure within $10 \%$ RF-modified adhesive specimens. (h) Small cracks within 5\% RF-modified adhesive specimens after 9 months of artificial saliva (arrows). (i) Cohesive failure in 10\% RF-modified adhesives after 9 months of storage. (j) Horizontal fracture seen at the interfacial region in 10\% RF-modified adhesive specimens after 9 months of storage. A, adhesive; RF, riboflavin. 
of the hybrid layer, especially in the control group (Figure 5a), which depicts a mixed failure pattern. In $1 \%$ RF-modified adhesive specimens, many open dentinal tubules can be observed on the debonded surfaces at the dentin side with partial coverage of the adhesive (Figure 5b), which denotes a predominantly mixed failure pattern similar to the control group. The failed specimens for 3\% RF-modified adhesive group (Figure $5 \mathrm{c}$ and $5 \mathrm{~d}$ ) presented islands of hybrid layer and resin tags and were in continuity with the dentin underneath. Several resin tags within the 3\% RF-modified adhesives occluded the dentinal tubules (Figure 5e). The 5\% RF-modified adhesive specimens also presented areas of mixed failure pattern and cohesive failure within the adhesive (Figure 5f). However, the 10\% RF-modified adhesive specimens presented primarily cohesive failures within the adhesive (Figure 5g).

After a 9-month storage in artificial saliva, similar fracture patterns were noted with the control, $1 \%$ and $3 \%$ RF-modified adhesive specimens similar to after the 24 -h storage. The 5\% RF-modified adhesive specimens demonstrated a mixed failure pattern after the 9-month storage (Figure 5h) similar to the 24-h storage; however, crack lines were characteristically observed among the adhesive layers. Furthermore, the 9-month 10\% RF-modified adhesive specimens showed a cohesive failure in the adhesive (Figure 5i). However, several of the $10 \%$ RF-modified adhesive specimens showed fractures or crack lines at the interface region (Figure $5 \mathrm{j}$ ).

DC

Table 3 shows the statistical analysis results of the DC for the control and RF-modified groups after $30 \mathrm{~min}$. Although the DC of the 3\% RFmodified specimens was numerically less, no significant difference was found between the control group and the 1\% and 3\% RF-modified adhesive groups. The 5\% RF and 10\% RF-modified specimens exhibited lower DC after photo-activation compared to the control, $1 \% \mathrm{RF}$ and 3\% RF-modified specimens $(P<0.05)$. Figure 6 shows the pattern of the degree of conversion starting from $10 \mathrm{~s}$ after the start of photoactivation until $30 \mathrm{~min}$ for all groups. A plateau was formed within 2-3 min for the control group and all of the experimental groups.

\section{Micro-Raman spectroscopy of dentin, control and modified adhesives}

Representative Raman spectra of the standard control adhesive (3M Single Bond), human dentin and resin-impregnated dentin recorded in the region of $800-1800 \mathrm{~cm}^{-1}$ are shown in Figure 2. The P-O stretching within the dentin mineral is identified at $960 \mathrm{~cm}^{-1}$ (Figure $2 \mathrm{~b}$ and $2 \mathrm{c}$ ). Additionally, several peaks are observed; from these, $1246 \mathrm{~cm}^{-1}, 1667 \mathrm{~cm}^{-1}$ and $1450 \mathrm{~cm}^{-1}$ are assigned to the Amide III, Amide ${ }^{36}$ and $\mathrm{C}-\mathrm{H}$ alkyl group, ${ }^{37}$ respectively. The $\mathrm{C}-\mathrm{H}$ alkyl group at $1450 \mathrm{~cm}^{-1}$ was selected as a measure of all of the organic components in the hybrid layer and as a measure of the resin-dentin adhesive penetration, similar to previous studies (Figure 2c).

Several peaks as the principal functional groups within all of the adhesive systems can be identified in Figure 7 as follows: $\mathrm{CH}_{2} \mathrm{CH}_{3}$ $1450 \mathrm{~cm}^{-1}$, phenyl $\mathrm{C}=\mathrm{C} 1610 \mathrm{~cm}^{-1}, \mathrm{C}=\mathrm{C} 1640 \mathrm{~cm}^{-1}$ and $\mathrm{C}=\mathrm{O}$
Degree of polymerization
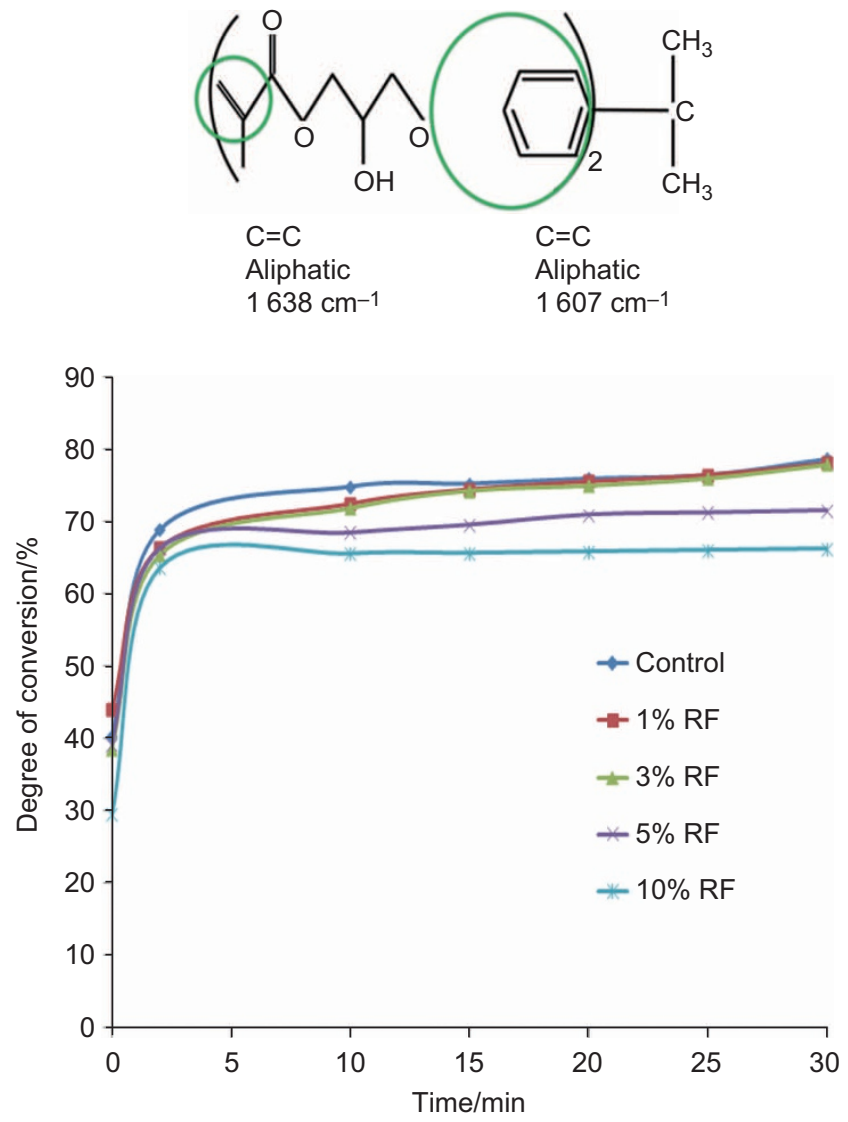

Figure 6 The percentage of degree of conversion of control and different RFmodified adhesive specimens at different time intervals from the start of photoactivation ( $0 \mathrm{~s})$ till $30 \mathrm{~min}$. RF, riboflavin.

$1720 \mathrm{~cm}^{-1}$. The $\mathrm{C}=\mathrm{O}$ peak for the different groups occurs as follows: control $=1717 \mathrm{~cm}^{-1}, 1 \%$ RF-modified adhesive $=1711 \mathrm{~cm}^{-1}, 3 \%$ RF-modified adhesives $=1710 \mathrm{~cm}^{-1}, 5 \%$ RF-modified adhesives $=1713 \mathrm{~cm}^{-1}$ and $10 \%$ RF-modified adhesives $=1727 \mathrm{~cm}^{-1}$. The molecules of aromatic and conjugated functional groups have delocalized electrons and are easily polarized due to the presence of double bonds or free electrons exhibiting increased Raman shifts. The rotation of molecules and transitions are easily observed within the absorption and scattering spectra. The Raman spectrum of dentin, the adhesive and resin dentin specimens have been recorded with low-power infra-red laser to eliminate the problems of sample fluorescence and photodecomposition. ${ }^{38}$

\section{Micro-Raman spectroscopy of resin-dentin specimens}

The spectrum represented at $1 \mu \mathrm{m}$ across the resin/dentin adhesive interface in all of the groups is shown in Figure 8 as a function of position across the resin/dentin interface. The Raman bands scanned across the resin-dentin interface at $960 \mathrm{~cm}^{-1}$ ( $\mathrm{P}-\mathrm{O}$ peak) and

Table 3 Means \pm standard deviations of degree of conversion of control and RF-modified dental adhesives

\begin{tabular}{|c|c|c|c|c|c|}
\hline Time & Control adhesive & $1 \%$ RF adhesive & $3 \%$ RF adhesive & $5 \%$ RF adhesive & $10 \%$ RF adhesive \\
\hline 30 min after light activation & $78.7 \pm(1.2)^{\mathrm{a}}$ & $78.1 \pm(1.7)^{a}$ & $77.9 \pm(1.5)^{\mathrm{a}}$ & $71.6 \pm(2.5)^{b}$ & $66.3 \pm(3.4)^{c}$ \\
\hline
\end{tabular}

RF, riboflavin.

Means followed by different letters differ statistically by Tukey's test $(P<0.05)$. Different letters (lower case row) indicate statistically significant difference $(P<0.05)$ 
$1450 \mathrm{~cm}^{-1}$ (C-H peak) are assigned to phosphate vibrations of hydroxyapatite and the $\mathrm{C}-\mathrm{H}$ alkyl group in the respective line maps (Figure 9). There was a gradual decrease in the $\mathrm{C}-\mathrm{H}$ band to the region of $14 \mu \mathrm{m}$. The depths achieved for the specimens were as follows: control, $11.6 \mu \mathrm{m} ; 1 \%$ RF-modified adhesive specimens, $14.1 \mu \mathrm{m} ; 3 \%$ RF-modified adhesive specimens, $13.1 \mu \mathrm{m}$; 5\% RF-modified adhesive specimens, $10.1 \mu \mathrm{m}$; and 10\% RF-modified adhesive specimens, $8.1 \mu \mathrm{m}$. From these line maps, the difference in the relative intensities of the $\mathrm{C}-\mathrm{H}$ alkyl peak started at a position of $3.9 \mu \mathrm{m}$ for the specimens and ended at $14 \mu \mathrm{m}$ for the $1 \%$ RF-modified adhesive. The 3\% RFmodified adhesive specimens also showed a difference in the relative intensity. However, there was a gradual decrease in the $\mathrm{C}-\mathrm{H}$ band to

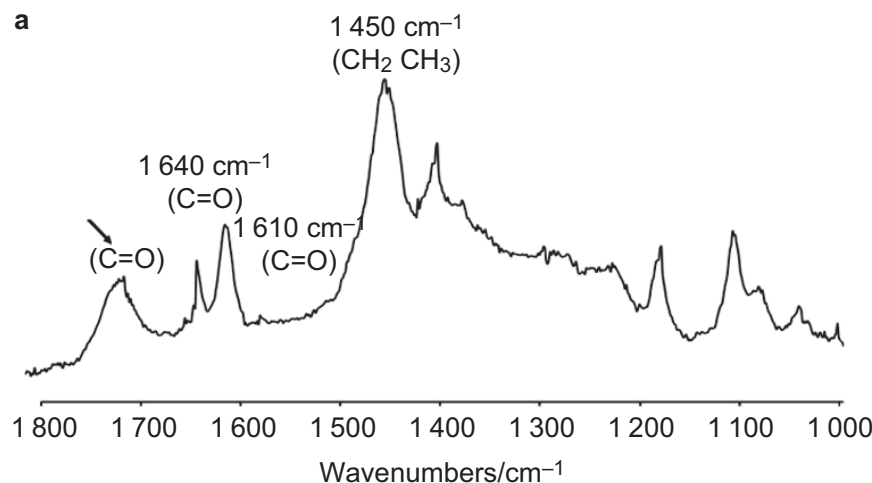

C

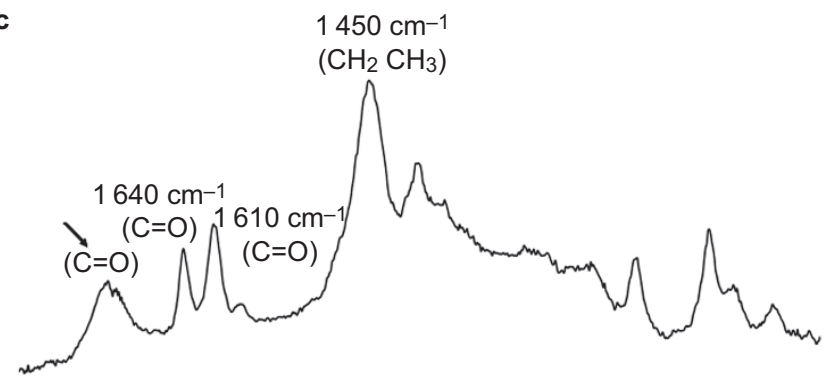

the region of $9 \mu \mathrm{m}$ after which it reached its plateau. These results indicated that the 3\% RF-modified adhesive specimen penetration was throughout more than $50 \%$ of the hybrid layer. The specimens showed a gradual plateau formation to the $13.1-\mu \mathrm{m}$ region representing good penetration for the monomer. The 5\% RF and 10\% RF-modified adhesive specimens showed the least amount of penetration and complete disassociation of the Raman spectrum at the Amide regions at 8-10 $\mu \mathrm{m}$. The micro-Raman data from the present study confirmed a complex interaction between the resin and the resin-dentin specimens.

The multiple Raman spectra were obtained in the control and RFmodified adhesive specimens at 5-8 $\mu \mathrm{m}$ levels within the hybrid layer. Figure 9 shows the Raman spectra at $5 \mu \mathrm{m}$ level in all of the groups

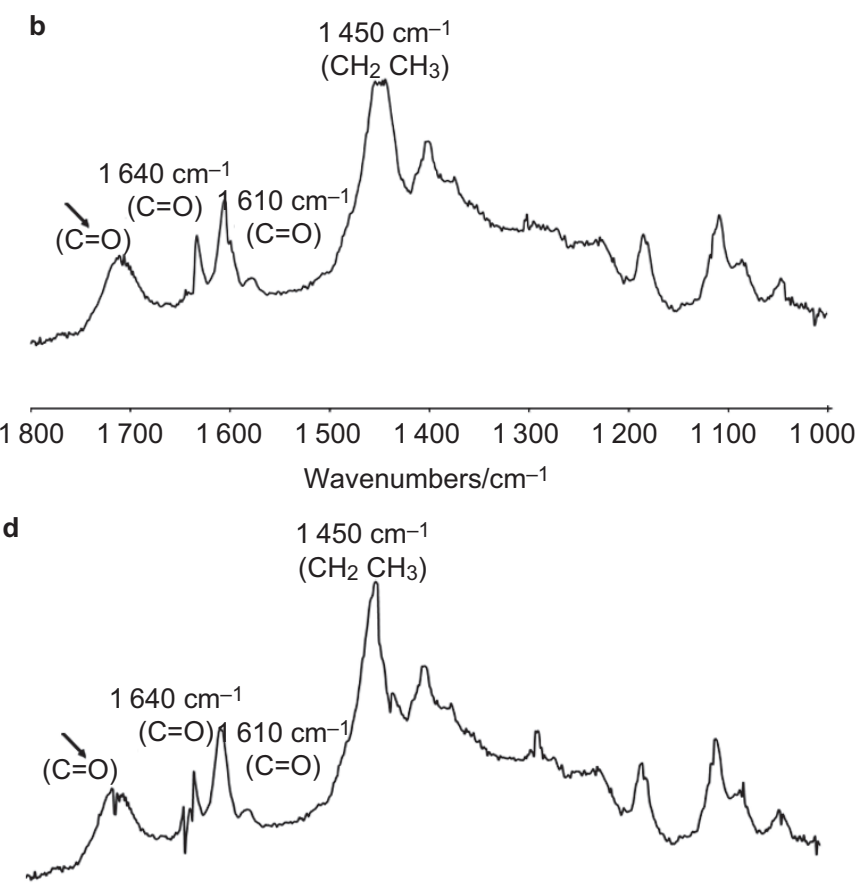

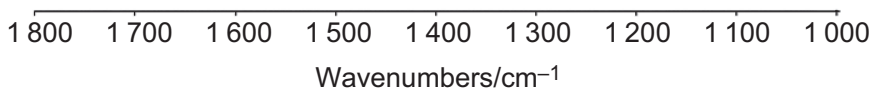
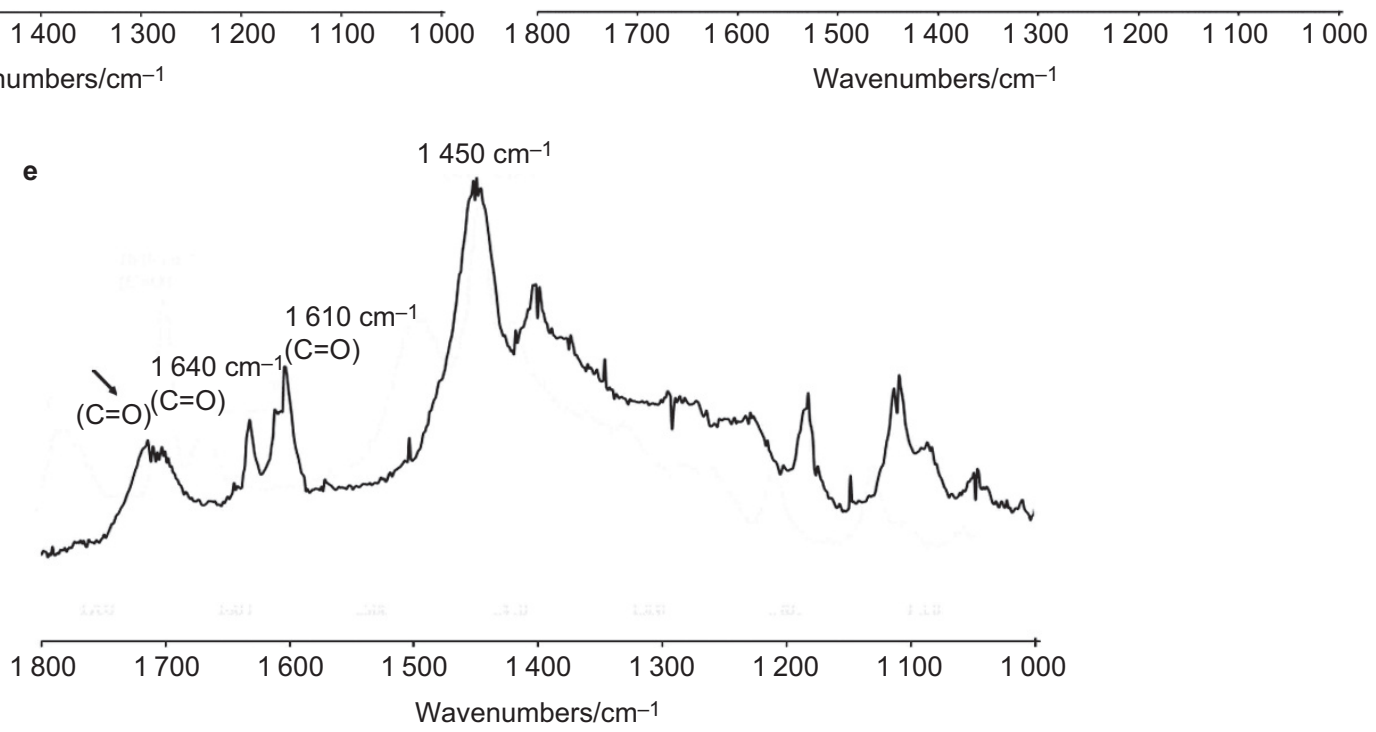

Figure 7 Representative Raman spectrum for the five adhesives tested illustrating the principal functional groups. The groups can be identified at $\mathrm{CH}_{2} \mathrm{CH}_{3}$ $1450 \mathrm{~cm}^{-1}, \mathrm{C}=01610 \mathrm{~cm}^{-1}, \mathrm{C}=01640 \mathrm{~cm}^{-1}$ and C=0 $1720 \mathrm{~cm}^{-1}$. The arrows indicate shifts associated with $\mathrm{C}=01720 \mathrm{~cm}^{-1} ;(\mathbf{a}) \mathrm{control}=1717 \mathrm{~cm}^{-1}$, (b) $1 \%$ RF-modified adhesive $=1711 \mathrm{~cm}^{-1}$, (c) $3 \%$ RF-modified adhesives $=1710 \mathrm{~cm}^{-1}$, (d) $5 \%$ RF-modified adhesives $=1713 \mathrm{~cm}^{-1}$ and (e) $10 \%$ RF-modified adhesives $=1727 \mathrm{~cm}^{-1}$. RF, riboflavin. 


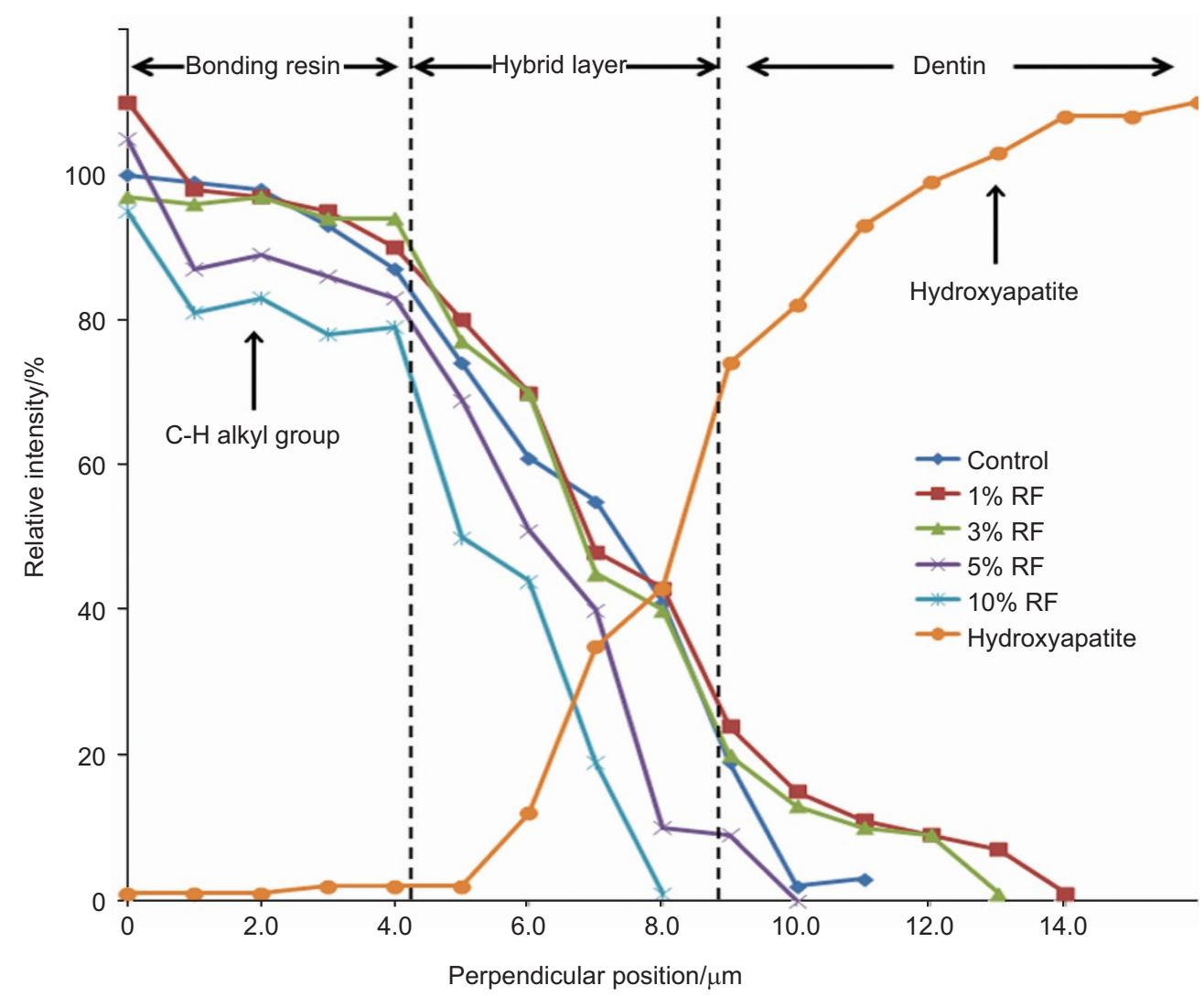

Figure 8 Representative line map (scans) across resin/dentin interface of different control and riboflavin-modified adhesive specimens. The spectral contribution is recorded at $960 \mathrm{~cm}^{-1}$ (hydroxyapatite) and $1450 \mathrm{~cm}^{-1}(\mathrm{C}-\mathrm{H}$ alkyl) intensities representing the penetration of different adhesives.

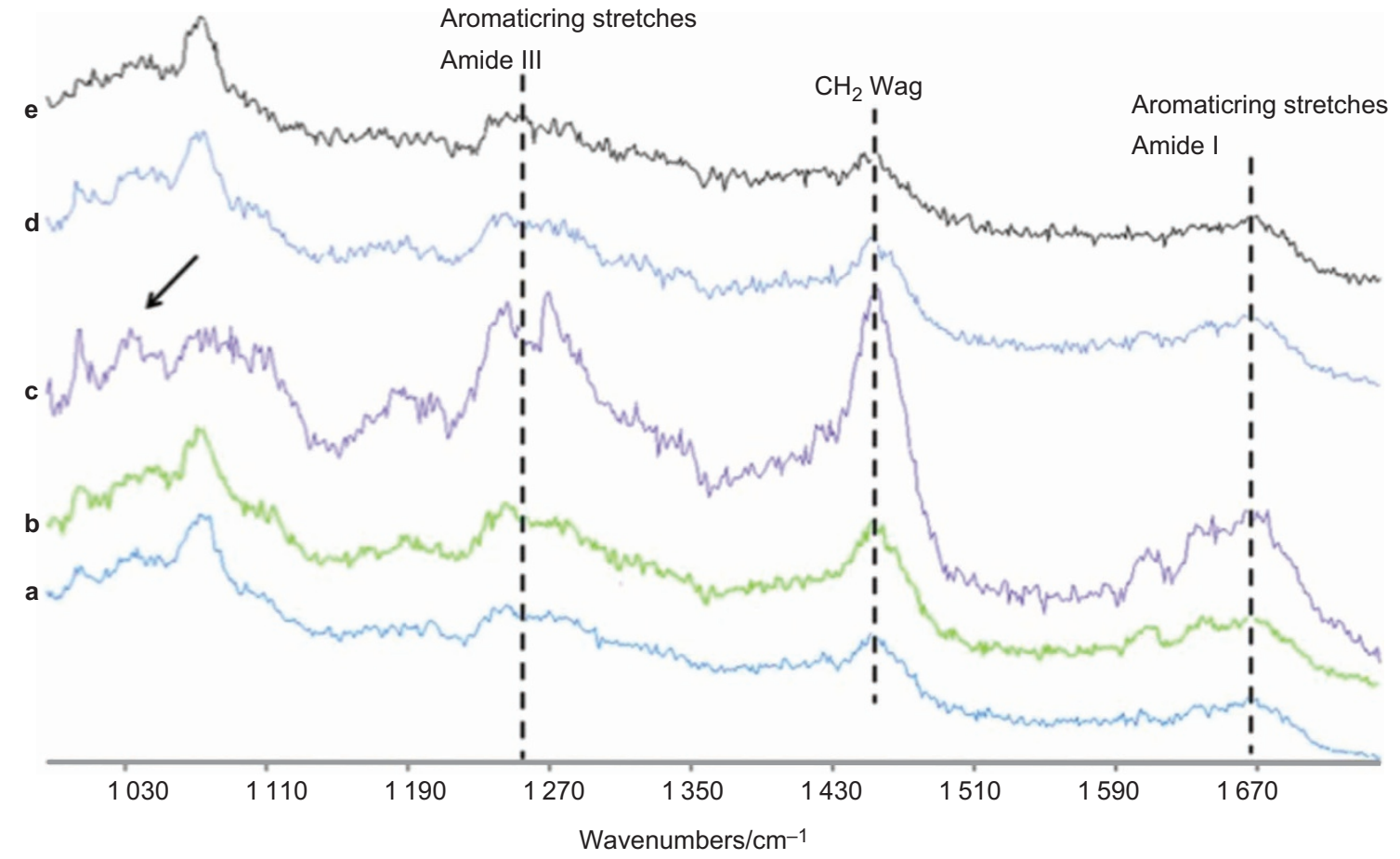

Figure 9 Comparison of Raman spectral data acquired in the region of $1030 \mathrm{~cm}^{-1}$ and $\mathbf{1} 670 \mathrm{~cm}^{-1}$ at $5 \mu \mathrm{m}$ levels. (a) Control; (b-e) $1 \%, 3 \%, 5 \%$ and $10 \% \mathrm{RF}$ modified adhesive specimens. The principal bands identified are amide bands (I and III) along with C-H alkyl groups in the resin-dentin specimens. The single arrow indicates the pyridinium ring group where accentuated intensity is observed in 3\% RF-modified adhesive specimen. RF, riboflavin. 
demonstrating primary band positions. The exceptions were noted in the Amide III and CH-alkyl groups of the 3\% RF-modified adhesive specimens where the Raman peaks were more accentuated compared to the other specimens. The Raman peaks observed at $5 \mu \mathrm{m}$ level in all of the groups demonstrated the following shifts for the specimens in the Amide I region: $1667 \mathrm{~cm}^{-1}, 1663 \mathrm{~cm}^{-1}, 1661 \mathrm{~cm}^{-1}, 1665 \mathrm{~cm}^{-1}$ and $1668 \mathrm{~cm}^{-1}$ for the control; $1 \%, 3 \%, 5 \%$ and $10 \%$ RF-modified adhesive specimens. The shifts observed in the Amide III region were noted as follows: $1246 \mathrm{~cm}^{-1}, 1249 \mathrm{~cm}^{-1}, 1253 \mathrm{~cm}^{-1}, 1248 \mathrm{~cm}^{-1}$ and $1247 \mathrm{~cm}^{-1}$ for the control; $1 \%, 3 \%, 5 \%$ and $10 \%$ RF-modified adhesive specimens. The pyridinium ring vibration at $1032 \mathrm{~cm}^{-1}$ showed no overlapping or remarkable changes except in the 3\% RFmodified adhesive specimens where the peak appeared to be slightly sharper (small arrow).

\section{DISCUSSION}

Ideally, the resin-dentin hybrid layer depicts a three-dimensional polymer/collagen network, enabling a stable link between the dentin and the adhesive; there is little evidence indicative of whether the ideal objective is achieved; ${ }^{39}$ instead, it has been conceived as the weakest link within the adhesive-dentin bond. ${ }^{40}$ The topical application of the primers or dental adhesives results in the flow inside the dentinal tubules and the interfibrillar spaces, which engulf the collagen fibrils with the resin monomers and provide a mechanical retention to the resin restorative. ${ }^{41}$ Additionally, a cascade of events has been hypothesized for the in vivo degradation of the hybrid layer due to vulnerability by the proteolytic enzymes to attack. ${ }^{42}$

Riboflavin, a known crosslinker, leads to strengthening of collagen fibrils through telopeptidase activity, which is a potent reduction of collagenase activity. ${ }^{43}$ The yellow moiety, RF, is responsible for the formation of singlet oxygen species, which instigates a crosslinking phenomenon within the collagen molecule. ${ }^{18}$ Furthermore, it is highly unstable under visible light; the behaviour has been interpreted to be unique in undergoing photo-reduction in the absence of an electron donor to form singlet oxygen. ${ }^{44}$ Previously, using a RF-based sensitizer has been reported to be favourable for reactions with tryptophan, tyrosine, histidine and methionine, which suggests that RF is an attractive crosslinker and a good oxidant. ${ }^{45}$

Previous studies have reported the significant positive effect of UVAactivated RF solutions on the mechanical and structural stability and the biodegradation resistance of dentin collagen matrix. Additionally, when applied as a dentin pre-treatment followed by photo-activation, RF has been reported to increase the bond strength by providing resistance to collagen degradation ${ }^{20}$ and to inactivate MMPs, particularly MMP-9. ${ }^{46}$ Moreover, our previous study suggested that the dental visible blue curing light could be considered to be a safer and efficient substitute compared to the UVA light sources for RF activation. ${ }^{21}$ Accordingly, in the present study, RF was incorporated as a direct component of experimental dentin adhesives, rather than as a separate pre-treatment as in previous studies, and the blue visible light was used for simultaneous photo-activation of RF and the photo-initiator/activator system of the experimental adhesive.

This study compared the bond strength durability, resin/dentin interface morphology and DC of the experimental, two-step, etchand-rinse RF-modified adhesives. The present study revealed appreciable differences in the resin/dentin interface morphology with the variation in RF content and storage time in artificial saliva (Figure 3). Additionally, the bonded specimens have provided bond strength values (Table 2) to dentin comparable with contemporary commercially available adhesive systems. ${ }^{47-49}$ Observing the SEM results at the resin/dentin interface revealed that the control, $1 \%$ and 3\% RF-modified adhesive specimens exhibited intact interfacial integrity after a 9-month storage (Figure $3 \mathrm{f}-3 \mathrm{~h}$ ), which might be favourably reflected by the high $\mu$ TBS values recorded, especially for the 3\% RF-modified adhesive specimens. In contrast, incorporating $10 \% \mathrm{RF}$ into the experimental adhesive specimen demonstrated a detrimental effect on the $\mu$ TBS values recorded at both $24 \mathrm{~h}$ and 9 months, which could be justified by the SEM images of the resin/dentin interface at 24-h (Figure 3e) and 9-month (Figure 3j) storage in artificial saliva.

From the results, the first null hypothesis can only be partially and conditionally accepted; with the increase in RF content up to $3 \%$, there was an increase in the $\mu \mathrm{TBS}$ in both the 24-h and 9month storage. However, with an additional increase in RF content up to $10 \%$, there was a significant decrease in $\mu$ TBS. These low $\mu$ TBS values recorded for $5 \%$ RF and $10 \%$ RF-modified adhesive specimens might be attributed to the overall increase in RF content inside the experimental adhesive. Additionally, the effect of RF on the polymerisation of the experimental dentin adhesive was evaluated as previously reported; RF has maximum absorption peaks at $270 \mathrm{~nm}, 366 \mathrm{~nm}$ and $445 \mathrm{~nm} \cdot{ }^{50-51}$ Accordingly, the competition between RF and the photo-activators/initiators of the dentin adhesive-system in the absorbing blue light might affect the polymerisation reaction of the resin monomers and should be investigated. From the results of the DC (Figure 6 and Table 3), the second null hypothesis could be rejected.

In our study, the incorporation of photo-oxidative RF increased $\mu$ TBS to a certain degree and then decreased so that the highest value of bond strength was obtained in the experimental adhesive containing $3 \%$ RF (Table 2). Concurrently, with an increase in the RF content up to $3 \% \mathrm{RF}$, no significant decrease in the DC was reported (Table 3). Moreover, when the RF content was increased up to $5 \%$ and $10 \%$, the degree of conversion was compromised, which could in part justify and explain the lower $\mu$ TBS values found with the higher RF content, especially $10 \%$. The blue light used in the study might have a dual effect in activating the RF and simultaneously in activating the polymerisation reaction of the resin monomers of the experimental adhesive. The increase in RF content could be suggested to induce its shielding effect against the blue light, ${ }^{18}$ especially at a higher concentration of RF. This correlation between the $\mu$ TBS and DC results can be supported with the SEM findings (Figure 3). Therefore, controlling the concentration of RF within the experimental adhesives used is crucial to achieve a compromise between the desirable effects of RF in crosslinking dentin collagen on one side and without compromising the degree of conversion and the polymerisation reaction of the dentin adhesive monomers. By adding RF to the experimental adhesive and simultaneous photoactivation, such combined steps might be less clinically complicated by decreasing the number of clinical steps required.

The qualitative SEM fracture analysis was performed to improve the accuracy of interpreting the results compared to the light microscopy and to facilitate the identification of the failure mode. ${ }^{52}$ The results of the fractographic analysis might also support and interpret the results of $\mu$ TBS and DC. As previously suggested, ${ }^{53}$ an increased number of mixed failures were related to increased bond strengths. For the failure mode distribution, the majority of the fractures demonstrated within immediate and 9-month storage specimens were mixed failures (Figures 4 and 5) in the control, 1\% and 3\% RF-modified adhesive specimens. In contrast, there was an increase in the cohesive failure within the adhesive with increasing RF content up to $5 \%$, with the highest among 10\% RF-modified adhesive specimens. This increase 
in the percentage of the cohesive failure in the adhesive might indicate the low mechanical properties of the $5 \%$ and $10 \%$ RF-modified experimental adhesive, which is in agreement with the low $\mu$ TBS (Table 2) and DC (Table 3) values reported.

The striking feature of spectra conducted for specimens is the absence of low fluorescence in the spectral peaks (Figures 2, 7 and 9). The use of $\mathrm{RF}$ as part of a two-step etch-and-rinse adhesive resulted in a change in the scattering intensity peaks within the organic phases specifically in the principal bands identified within the Amide band region along with $\mathrm{C}-\mathrm{H}$ alkyl groups in the resin/dentin interface specimens. The Raman peaks at $1450 \mathrm{~cm}^{-1}$ correspond to the $\mathrm{CH}_{2} \mathrm{CH}_{3}$ present in most monomers and are indicative of the resin penetration within the dentin specimens. The present study is not an attempt to identify the known compounds within the adhesive; the present study aims to analyse the change within the known Raman peak positions along the depth of the resin/dentin interface. According to our previous study, RF was sufficient for the formation of crosslinking bonds in the collagen using Raman spectroscopy. A peptide bond within the collagen molecule is able to give rise to vibrational modes, such as the Amide regions (Amide I and III) and are clearly detected in the Raman spectra (Figures 2 and 9). When the dentin is restored using RF-modified adhesives, the shifts within the Raman peaks at $5 \mu \mathrm{m}$ suggest an increase in Amide III band frequency and a decrease in Amide I band frequency (Figure 9). These Amide bands indicate stability of the collagen structure as a result of hydrogen bonding between prolines and glycines. ${ }^{54}$ These spectral alterations can be suggestive of changes in the protein structure as a result of the RF content within the adhesive. Previously, McCall et al. ${ }^{55}$ explained the interactions of RF-UVA using collagen in the human cornea and the formation of crosslinks within the collagen structure as a result of the oxygen radicals produced. ${ }^{55}$ The significant finding of the organic phase in the region of $1030 \mathrm{~cm}^{-1}$ to $1700 \mathrm{~cm}^{-1}$ had been presented. The peaks at $\mathrm{C}=\mathrm{H}$ bending mode in the region of $1450 \mathrm{~cm}^{-1}$ in the spectra of specimens in different RF-modified adhesive specimens confirm the impregnation of resin at variant levels according to the concentration of RF used within the experimental adhesive formulation. A comparison of the spectra of $\mathrm{C}=\mathrm{H}$ bending mode among all of the specimens showed a higher intensity for 3\% RFmodified adhesive specimen (data acquired at $5 \mu \mathrm{m}$ level) due to the $\mathrm{C}=\mathrm{H}$ bending mode that had been subjected to interference. The interference was attributed to the crosslinking effect of the RF, which indicated a sharp well-defined band at $1452 \mathrm{~cm}^{-1}\left(\mathrm{CH}_{2}\right.$ wag) for $3 \% \mathrm{RF}-$ modified adhesive specimen that appeared at a lower intensity among other specimens. Comparing the spectra of different specimens indicated that there had been significant structural changes from the spectrum of standard control dentin tissue and the modified experimental adhesives within the principal functional groups providing a baseline. There was a net effect with an increase in the signal/noise ratio of the organic vibrational bands. The small apparent shifts within the Raman bands of Amide I and III region were well within the estimated error of measurement, although small amounts of fluorescence remained after the RF-modified adhesive specimens. The magnitude of shifts after the crosslinking effect of dentin suggested that there had been a structural perturbation in the collagen matrix.

Interestingly, the changes within the Raman spectrum are noted among the adhesive specimens containing RF. Additionally, from the results, the peak at $1032 \mathrm{~cm}^{-1}$ provides an important method for verifying the effect of collagen and the crosslinking agents. ${ }^{56}$ Raman spectroscopy is considered to be important in analysing variations and interactions of complex molecules, such as a measure of direct effect of the crosslinkers on dentin collagen fibrils in the resin-dentin bonding. The changes were specifically analysed within the pyridinium ring group where accentuated intensity was observed in 3\% RF-modified adhesive specimen and changes within the $1450 \mathrm{~cm}^{-1}$, which is the $\mathrm{C}=\mathrm{H}$ bending mode. The inorganic phase within the dentin structure and the resin dentin specimens had substantially remained unaffected. The common example had been set to the phosphate moiety, which was centred on $958-960 \mathrm{~cm}^{-1}\left(\mathrm{PO}_{4}{ }^{3-}\right.$ stretching mode). ${ }^{38}$ The peak at approximately $584 \mathrm{~cm}^{-1}\left(\mathrm{PO}_{4}{ }^{3-}\right)$ was also observed and calculated to identify its static reading and was noted to remain unchanged. The data had been collected in a concentrated fashion, such as Amide III at $1242 \mathrm{~cm}^{-1}$; the peak at $1262 \mathrm{~cm}^{-1}$ was not considered. Therefore, the alterations in the positions of the Raman bands for the RF-modified adhesive specimens were indicative of the crosslinking.

The changes within the representative line map (Figure 8) across the resin/dentin interface can be observed in different groups. From the results of the study, $1 \%$ and $3 \% \mathrm{RF}$ adhesive specimens showed higher amounts of penetration compared to the control, 5\% and $10 \%$ RFmodified specimens. The penetration depths for the different specimens seemed to be dependent on the RF concentration within the adhesive. The penetration of $1 \% \mathrm{RF}$ adhesive was higher than 3\% (Figure 8 ), which indicated a higher polymerisation of the $1 \%$ RF-modified adhesive in decalcified dentin. There was likely a competition between the RF and the photo-initiators of the dentin-adhesive system in absorbing the blue light, which might affect the polymerisation reaction of the adhesive system used. Subsequently, with an increase in RF content up to 3\%, there was no substantial increase in the penetration depths. It was only when the RF concentration within the adhesive was increased to $5 \%$ and $10 \%$ that the penetration depths were compromised to a greater extent, which might suggest that the lower penetration values and the dual effect of blue light activate the RF and the photo-initiator system simultaneously within the adhesive. Table 3 shows the statistical analysis results of the penetration depths for the control and the RF-modified groups. The penetration depths of the 3\% RF-modified adhesive specimens were slightly less than the control group. However, no significant difference was found between the control group and the $1 \%$ and $3 \%$ RF-modified adhesive specimens. Concurrently, with the increase in RF content up to $3 \%$, no significant decrease in penetration depths was reported. However, when the RF was increased up to $5 \%$ and $10 \%$, the penetration depth was compromised further, which may justify and explain the depth of cure. Consequently, the null hypothesis can be rejected because the RF incorporation did affect the resin penetration of the modified adhesive.

Including RF within the adhesive may provide a long-term solution of crosslinking the dentin matrix collagen and may make it easier for clinicians to compare the results with pre-treatment procedures. Any pre-treatment procedure can be uncontrolled, and it is difficult to determine the crosslinking level achieved. Additionally, the inactivation effect of the crosslinking agent may be lessened after acid etching once the rinse water removes the crosslinking solution before treatment. Consequently, the hybrid layers that were created from the twostep etch-and-rinse adhesives degrade over time both in vitro and in vivo. This degradation is due to the loss of cross-banding of the collagen fibrils that results from increased water degradation and MMP2/MMP-9 gelatinase activity. ${ }^{57}$ We can speculate that RF can inactivate MMPs and may be explained by a reduction of MMP activities due to the direct MMP crosslinking ${ }^{12}$ and due to the development of an intricate collagen fibrillar network mentioned in our previous study that may facilitate in better adhesion. Therefore, incorporating RF within the two-step etch-and-rinse adhesive can be considered to be 
a prudent and advisable approach for achieving the desirable effects of RF in crosslinking the dentin collagen fibrillar network at a time when the dentin is subjected to etching, which activates endogenous MMPs. ${ }^{58}$ In the present study, incorporating RF within the adhesive is a unique approach rather than using RF as a separate pre-treatment, as reported in previous studies. The blue visible light was used for dual photo-activation of RF and the photo-initiator system of the adhesive. The results of the RF-modified resin dentin and adhesive specimens clearly indicate the beneficial use of Raman spectroscopy, which allows detailed spectral analysis as well as quality of structural and compositional detail and minimal sample preparation. The collagen matrix characterisation after RF crosslinking should continue to enhance the vibrational spectra of phosphate minerals for the purpose of identification and characterisation.

\section{CONCLUSIONS}

Considering the experimental conditions of this in vitro study and the results achieved, we can conclude that the incorporation of RF in the experimental two-step etch-and-rinse adhesive at 3\% improved the immediate bond strength and bond durability after a 9-month storage in artificial saliva without adversely affecting the degree of conversion in the adhesive monomers. There was no significant difference between the control group and the $1 \%$ and 3\% RF-modified adhesive groups. However, additional studies should be performed to investigate the detailed effects of RF on the dentin collagen network within the hybrid layer formed and to assess the stability of the demineralized dentin collagen matrix.

\section{ACKNOWLEDGEMENTS}

This work was supported, in part, by the Ministry of Education, Singapore, NUS/ARF grants R221000039133 and R221000052112.

1 Tay FR, Pashley DH. Dental adhesives of the future. J Adhes Dent 2002; 4(2): 91-103.

2 Vargas MA, Cobb DS, Denehy GE. Interfacial micromorphology and shear bond strength of single-bottle primer/adhesives. Dent Mater 1997; 13(5): 316-324.

3 Nakabayashi N, Ashizawa M, Nakamura M. Identification of a resin-dentin hybrid layer in vital human dentin created in vivo: durable bonding to vital dentin. Quintessence Int 1992; 23(2): 135-141.

4 Breschi L, Mazzoni A, Nato F et al. Chlorhexidine stabilizes the adhesive interface: a 2-year in vitro study. Dent Mater 2010; 26(4): 320-325.

5 Wang $Y$, Spencer $P$. Hybridization efficiency of the adhesive/dentin interface with wet bonding. J Dent Res 2003; 82(2): 141-145.

6 Van Meerbeek B, Yoshihara K, Yoshida Y et al. State of the art of self-etch adhesives. Dent Mater 2011; 27(1): 17-28.

7 Nishitani Y, Yoshiyama M, Wadgaonkar B et al. Activation of gelatinolytic/collagenolytic activity in dentin by self-etching adhesives. Eur J Oral Sci 2006; 114(2): 160-166.

8 Fang M, Liu R, Xiao Y et al. Biomodification to dentin by a natural crosslinker improved the resin-dentin bonds. J Dent 2012; 40(6): 458-466.

9 Fratzl P, Gupta H, Paschalis E et al. Structure and mechanical quality of the collagenmineral nano-composite in bone. J Mater Chem 2004; 14(1): 2115-2123.

10 Gelse K, Pöschl E, Aigner T. Collagens-structure, function, and biosynthesis. Adv Drug Deliv Rev 2003; 55(12): 1531-1546.

11 Bedran-Russo AK, Yoo KJ, Ema KC et al. Mechanical properties of tannic-acid-treated dentin matrix. J Dent Res 2009; 88(9): 807-811.

12 Tezvergil-Mutluay A, Agee KA, Hoshika T et al. The requirement of zinc and calcium ions for functional MMP activity in demineralized dentin matrices. Dent Mater 2010; 26(11): 1059-1067.

13 Wallace DG, Rosenblatt J. Collagen gel systems for sustained delivery and tissue engineering. Adv Drug Deliv Rev 2003; 55(12): 1631-1649.

14 Han B, Jaurequi J, Tang BW et al. Proanthocyanidin: a natural crosslinking reagent for stabilizing collagen matrices. J Biomed Mater Res A 2003; 65(1): 118-124.

15 Choe E, Min DB. Chemistry and reactions of reactive oxygen species in foods. Crit Rev Food Sci Nutr 2006; 46(1): 1-22.

16 Slifkin MA. Interaction of amino-acids with riboflavin. Nature 1963; 197: 275-276.

17 Matheson IB, Etheridge RD, Kratowich NR et al. The quenching of singlet oxygen by amino acids and proteins. Photochem Photobiol 1975; 21(3): 165-171.

18 Snibson GR. Collagen cross-linking: a new treatment paradigm in corneal disease -a review. Clin Experiment Ophthalmol 2010; 38(2): 141-153.
19 Wollensak G, Spoerl E, Seiler T. Riboflavin/ultraviolet-A-induced collagen crosslinking for the treatment of keratoconus. Am J Ophthalmol 2003; 135(5): 620-627.

20 Fawzy A, Nitisusanta L, Iqbal $\mathrm{K}$ et al. Characterization of riboflavin-modified dentin collagen matrix. J Dent Res 2012; 91(11): 1049-1054.

21 Fawzy AS, Nitisusanta LI, Iqbal $\mathrm{K}$ et al. Riboflavin as a dentin crosslinking agent: ultraviolet A versus blue light. Dent Mater 2012; 28(12): 1284-1291.

22 Bellamy LJ. The infrared spectra of complex molecules. Vol. 1, 3rd ed. London: Chapman and Hall, 1975: 37-148.

23 Spencer $\mathrm{P}$, Wang Y, Walker MP et al. Interfacial chemistry of the dentin/adhesive bond. J Dent Res 2000; 79(7): 1458-1463.

24 Wieliczka DM, Kruger MB, Spencer P. Raman imaging of dental adhesive diffusion. App/ Spect 1997; 51: 1593-1596.

25 Van Meerbeek B, Mohrbacher H, Celis JP et al. Chemical characterization of the resindentin interface by micro-Raman spectroscopy. J Dent Res 1993; 72(10): 14231428.

26 Hanlon EB, Manoharan R, Koo TW et al. Prospects for in vivo Raman spectroscopy. Phys Med Biol 2000; 45(2): R1-R59.

$27 \mathrm{Ye} Q$, Spencer $\mathrm{P}$, Wang $\mathrm{Y}$ et al. Relationship of solvent to the photopolymerization process, properties, and structure in model dentin adhesives. J Biomed Mater Res A 2007; 80(2): 342-350.

28 Park J, Ye Q, Topp EM et al. Effect of photoinitiator system and water content on dynamic mechanical properties of a light-cured bisGMA/HEMA dental resin. J Biomed Mater Res A 2010; 93(4): 1245-1251.

29 Arı H, Dönmez N, Belli S. Effect of artificial saliva contamination on bond strength to pulp chamber dentin. Eur J Dent 2008; 2(2): 86-90.

30 Heymann HO, Bayne SC. Current concepts in dentin bonding: focusing on dentinal adhesion factors. J Am Dent Assoc 1993; 124(5): 26-36.

31 Epasinghe DJ, Yiu CK, Burrow MF et al. Effect of proanthocyanidin incorporation into dental adhesive resin on resin-dentine bond strength. J Dent 2012; 40(3): 173-180.

32 Yoshida T, Yamaguchi K, Tsubota $\mathrm{K}$ et al. Effect of metal conditioners on polymerization behavior of bonding agents. J Oral Sci 2005; 47(4): 171-175.

33 Heigl JJ, Bell MF, White JN. Application of infrared spectroscopy to the analysis of liquid hydrocarbons. Anal Chem 1947; 19(5): 293-298.

34 Jose $\mathrm{P}$, Sakhamuri S, Sampath $\mathrm{V}$ et al. Degree of conversion of two dentin bonding agents with and without a desensitizing agent using fourier transform infrared spectroscopy: an in vitro study. J Conserv Dent 2011; 14(3): 302-305.

$35 \mathrm{Xu} \mathrm{C}$, Yao X, Walker MP et al. Chemical/molecular structure of the dentin-ename junction is dependent on the intratooth location. Calcif Tissue Int 2009; 84(3): 221-228.

$36 \mathrm{Xu} \mathrm{C}$, Wang Y. Collagen cross linking increases its biodegradation resistance in wet dentin bonding. J Adhes Dent 2012; 14(1): 11-18.

37 Ozaki M, Suzuki M, Itoh $\mathrm{K}$ et al. Laser-Raman spectroscopic study of the adhesive interface: analysis between 4-META/MMA-TBB resin and bovine or human dentin. Dent Mater J 1992; 11(1): 70-76.

38 Rehman I, Smith R, Hench LL et al. Structural evaluation of human and sheep bone and comparison with synthetic hydroxyapatite by FT-Raman spectroscopy. J Biomed Mater Res 1995; 29(10): 1287-1294.

39 Wang $Y$, Spencer $P$. Continuing etching of an all-in-one adhesive in wet dentin tubules. J Dent Res 2005; 84(4): 350-354.

40 Sano H, Yoshikawa T, Pereira PN et al. Long-term durability of dentin bonds made with a self-etching primer, in vivo. J Dent Res 1999; 78(4): 906-911.

41 Sano H, Takatsu T, Ciucchi B et al. Tensile properties of resin-infiltrated demineralized human dentin. J Dent Res 1995; 74(4): 1093-1102.

42 de Munck J, Van Landuyt K, Peumans M et al. A critical review of the durability of adhesion to tooth tissue: methods and results. J Dent Res 2005; 84(2): 118132.

43 Okada Y, Naka K, Kawamura K et al. Localization of matrix metalloproteinase 9 (92kilodalton gelatinase/type IV collagenase $=$ gelatinase $\mathrm{B}$ ) in osteoclasts: implications for bone resorption. Lab Invest 1995; 72(3): 311-322.

44 Oster G, Holmstrom B. Riboflavin as an electron donor in photochemical reactions. J Am Chem Soc 1961; 83(8): 1867-1871.

45 Edwards AM, Silva E. Effect of visible light on selected enzymes, vitamins and amino acids. J Photochem Photobiol B 2001; 63(1/2/3): 126-131.

46 Cova A, Breschi L, Nato F et al. Effect of UVA-activated riboflavin on dentin bonding. J Dent Res 2011; 90(12): 1439-1445.

47 Sadek FT, Goracci C, Cardoso PE et al. Microtensile bond strength of current dentin adhesives measured immediately and 24 hours after application. J Adhes Dent 2005; 7(4): 297-302.

48 Salvio LA, Hipólito VD, Martins AL et al. Hybridization quality and bond strength of adhesive systems according to interaction with dentin. Eur J Dent 2013; 7(3): 315326.

49 Jose $\mathrm{P}$, Sakhamuri S, Sampath $\mathrm{V}$ et al. Degree of conversion of two dentin bonding agents with and without a desensitizing agent using Fourier transform infrared spectroscopy: an in vitro study. J Conserv Dent 2011; 14(3): 302-305.

50 Kohlhaas M, Spoerl E, Schilde T et al. Biomechanical evidence of the distribution of cross-links in corneas treated with riboflavin and ultraviolet A light. J Cataract Refract Surg 2006; 32(2): 279-283.

51 Spoerl E, Mrochen M, Sliney D et al. Safety of UVA-riboflavin cross-linking of the cornea. Cornea 2007; 26(4): 385-389.

52 Hashimoto M, Ohno H, Sano H et al. In vitro degradation of resin-dentin bonds analyzed by microtensile bond test, scanning and transmission electron microscopy. Biomaterials 2003; 24(21): 3795-3803. 
53 Osorio R, Pisani-Proenca J, Erhardt MC et al. Resistance of ten contemporary adhesives to resin-dentine bond degradation. J Dent 2008; 36(2): 163-169.

54 Fraser RD, MacRae TP, Suzuki E. Chain conformation in the collagen molecule. J Mol Biol 1979; 129(3): 463-481.

55 McCall AS, Kraft S, Edelhauser HF et al. Mechanisms of corneal tissue cross-linking in response to treatment with topical riboflavin and long-wavelength ultraviolet radiation (UVA). Invest Ophthalmol Vis Sci 2010; 51(1): 129-138.

$56 \mathrm{Xu}$ C, Wang Y. Cross-linked demineralized dentin maintains its mechanical stability when challenged by bacterial collagenase. J Biomed Mater Res Part B Appl Biomater 2011; 96(2): 242-248.

57 Carrilho MR, Geraldeli S, Tay F et al. In vivo preservation of the hybrid layer by chlorhexidine. J Dent Res 2007; 86(6): 529-533.
58 Nakabayashi N, Pashley DH. Hybridization of dental hard tissues. Quint Pub 1998; 1: 65-73.

cc) (i) $\Theta$ This work is licensed under a Creative Commons Attribution-

cc) NonCommercial-NoDerivs 3.0 Unported License. The images or other third party material in this article are included in the article's Creative Commons license, unles indicated otherwise in the credit line; if the material is not included under the Creative Commons license, users will need to obtain permission from the license holder to reproduce the material. To view a copy of this license, visit http://creativecommons.org/licenses/ by-nc-nd/3.0/ 